\title{
Multimolecule ALMA observations toward the Seyfert 1 galaxy NGC 1097
}

S. Martín ${ }^{1}$, K. Kohno ${ }^{2,3}$, T. Izumi ${ }^{2}$, M. Krips ${ }^{1}$, D. S. Meier ${ }^{4,5}$, R. Aladro ${ }^{6}$, S. Matsushita ${ }^{7}$, S. Takano ${ }^{8,9}$, J. L. Turner ${ }^{10}$, D. Espada ${ }^{11,12,13}$, T. Nakajima ${ }^{14}$, Y. Terashima ${ }^{15}$, K. Fathi ${ }^{16,17}$, P.-Y. Hsieh ${ }^{7,18}$, M. Imanishi ${ }^{13,19}$, A. Lundgren ${ }^{11}$, N. Nakai ${ }^{20}$, E. Schinnerer ${ }^{21}$, K. Sheth ${ }^{22}$, and T. Wiklind ${ }^{11}$

1 Institut de Radio Astronomie Millimétrique, 300 rue de la Piscine, Dom. Univ., 38406 St Martin d'Hères, France e-mail: smartin@iram.fr

2 Institute of Astronomy, The University of Tokyo, 2-21-1 Osawa, Mitaka, 181-0015 Tokyo, Japan

3 Research Center for the Early Universe, The University of Tokyo, 7-3-1 Hongo, Bunkyo, 113-0033 Tokyo, Japan

4 New Mexico Institute of Mining and Technology, 801 Leroy Place, Socorro, NM, USA

5 National Radio Astronomy Observatory, Pete V. Domenici Array Science Center, PO Box O, Socorro, NM, 87801, USA

${ }^{6}$ European Southern Observatory, Alonso de Córdova 3107, Vitacura, Casilla 19001 Santiago 19, Chile

7 Academia Sinica, Institute of Astronomy \& Astrophysics, PO Box 23-141, 10617 Taipei, Taiwan

8 Nobeyama Radio Observatory, Nobeyama, Minamimaki, Minamisaku, 384-1305 Nagano, Japan

9 Department of Astronomical Science, The Graduate University for Advanced Studies (Sokendai), Nobeyama, Minamimaki, Minamisaku, 384-1305 Nagano, Japan

${ }^{10}$ Department of Physics and Astronomy, UCLA, 430 Portola Plaza, Los Angeles, CA 90095-1547, USA

11 Joint ALMA Observatory (JAO), 3107 Alonso de Córdova, Vitacura, Santiago, Chile

12 National Astronomical Observatory of Japan (NAOJ), 2-21-1 Osawa, Mitaka, 181-8588, Tokyo, Japan

13 Department of Astronomical Science, The Graduate University for Advanced Studies (SOKENDAI), 2-21-1 Osawa, Mitaka, 181-8588 Tokyo, Japan

14 The Solar-Terrestrial Environment Laboratory, Nagoya University, Furo-cho, Chikusa-ku, Nagoya, 464-8601 Aichi, Japan

15 Department of Physics, Ehime University, 2-5 Bunkyo-cho, Matsuyama, 790-8577 Ehime, Japan

16 Stockholm Observatory, Department of Astronomy, Stockholm University, AlbaNova Centre, 10691 Stockholm, Sweden

17 Oskar Klein Centre for Cosmoparticle Physics, Stockholm University, 10691 Stockholm, Sweden

18 Institute of Astronomy, National Central University, No. 300, Jhongda Road, Jhongli City, 32001 Taoyuan County, Taiwan

19 Subaru Telescope, National Astronomical Observatory of Japan, 650 North A'ohoku Place, Hilo, HI 96720, USA

${ }^{20}$ Division of Physics, Faculty of Pure and Applied Science, University of Tsukuba, Tsukuba, 305-8571 Ibaraki, Japan

21 Max Planck Institute for Astronomy, Königstuhl 17, 69117 Heidelberg, Germany

22 National Radio Astronomy Observatory, 520 Edgemont Road, Charlottesville, VA 22903, USA

Received 3 October 2014 / Accepted 10 October 2014

\section{ABSTRACT}

Context. The nearby Sy 1 galaxy NGC 1097 represents an ideal laboratory for exploring the molecular chemistry in the surroundings of an active galactic nucleus (AGN).

Aims. Exploring the distribution of different molecular species allows us to understand the physical processes affecting the interstellar medium both in the AGN vicinity and in the outer star forming molecular ring.

Methods. We carried out $3 \mathrm{~mm}$ ALMA observations that include seven different molecular species, namely $\mathrm{HCN}, \mathrm{HCO}+\mathrm{CCH}^{+} \mathrm{CS}$, $\mathrm{HNCO}, \mathrm{SiO}, \mathrm{HC}_{3} \mathrm{~N}$, and $\mathrm{SO}$, as well as the ${ }^{13} \mathrm{C}$ isotopologues of the first two. Spectra were extracted from selected positions and all species were imaged over the central $2 \mathrm{kpc}\left(\sim 30^{\prime \prime}\right)$ of the galaxy at a resolution of $\sim 2.2^{\prime \prime} \times 1.5^{\prime \prime}(150 \mathrm{pc} \times 100 \mathrm{pc})$.

Results. $\mathrm{HCO}^{+}$and CS appear to be slightly enhanced in the star forming ring. $\mathrm{CCH}$ shows the largest variations across NGC 1097 and is suggested to be a good tracer of both obscured and early stage star formation. $\mathrm{HNCO}, \mathrm{SiO}$, and $\mathrm{HC}_{3} \mathrm{~N}$ are significantly enhanced in the inner circumnuclear disk surrounding the AGN.

Conclusions. Differences in the molecular abundances are observed between the star forming ring and the inner circumnuclear disk. We conclude that the $\mathrm{HCN} / \mathrm{HCO}^{+}$and $\mathrm{HCN} / \mathrm{CS}$ differences observed between AGN-dominated and starburst (SB) galaxies are not due to a $\mathrm{HCN}$ enhancement due to X-rays, but rather this enhancement is produced by shocked material at distances of $200 \mathrm{pc}$ from the AGN. Additionally, we claim that lower HCN/CS is a combination of a small underabundance of CS in AGNs, together with excitation effects, where a high density gas component $\left(\sim 10^{6} \mathrm{~cm}^{-3}\right)$ may be more prominent in SB galaxies. However, the most promising are the differences found among the dense gas tracers that, at our modest spatial resolution, seem to outline the physical structure of the molecular disk around the AGN. In this picture, $\mathrm{HNCO}$ probes the well-shielded gas in the disk, surrounding the dense material moderately exposed to the X-ray radiation traced by $\mathrm{HC}_{3} \mathrm{~N}$. Finally $\mathrm{SiO}$ might be the innermost molecule in the disk structure.

Key words. astrochemistry - ISM: abundances - ISM: molecules - galaxies: ISM

\section{Introduction}

The molecular material in galaxies is a fundamental ingredient in the fueling of the energetic phenomena in galactic nuclei.
Though the mass and distribution of the molecular gas can be probed by the bright lines of carbon monoxide, deep observations of fainter molecular species have been proven as excellent tools of the physical processes affecting the molecular gas. This 


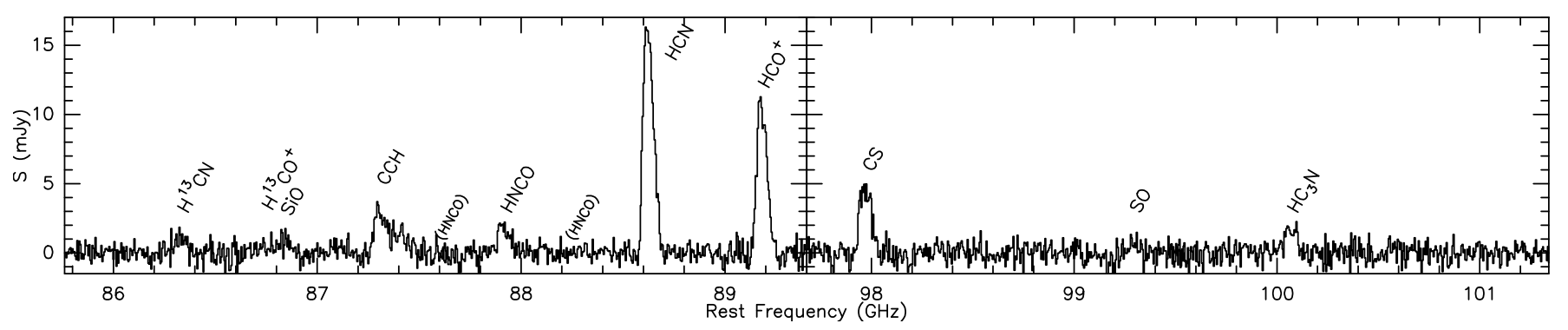

Fig. 1. Full spectral coverage of the ALMA Band 3 observations extracted from the nuclear position of NGC 1097 where the peak of molecular emission is observed for all species. This position corresponds to position A in Table 2 and Fig. 2.

has been illustrated by numerous deep spectral line surveys during the last decade (see review by Martín 2011), as well as extensive work on the theoretical modeling (e.g., Meijerink et al. 2007; Bayet et al. 2008, 2011; Loenen et al. 2008; Meijerink et al. 2013; Harada et al. 2010, 2013). The molecular chemistry in the interstellar medium (ISM) provides direct information on the environmental conditions and therefore on the type of activity in the central few hundred parsecs of galaxies. Moreover, the observation of individual molecular species allow us not only to estimate the physical conditions, but also to dissect the ISM into the different heating mechanisms in play as observed within the central molecular zone of our own Galaxy (Amo-Baladrón et al. 2011; Jones et al. 2012; Martín et al. 2012).

Most of the deep and/or unbiased chemical studies in galaxies have been carried out at very low resolution (i.e., $\gg 100 \mathrm{pc}$, Wang et al. 2004; Martín et al. 2006, 2011; Aladro et al. 2011a, 2013; Davis et al. 2013; Watanabe et al. 2014) but for absorption studies toward bright continuum high- $z$ sources where, though extremely prolific in molecular detection, no spatial information can be obtained (Muller et al. 2011, 2014). High resolution studies toward extragalactic sources have mostly focused toward the brightest nearby galaxies, as well as on the brightest species after carbon monoxide: $\mathrm{HCN}$ and $\mathrm{HCO}^{+}$(NGC 253 at $3^{\prime \prime}$ resolution, Knudsen et al. (2007); M51 at 4" , Schinnerer et al. 2010; NGC 1068 at 1", Krips et al. 2011; NGC 1097 at 3", Hsieh et al. 2012; or a sample of luminous infrarred galaxies (LIRGs) at $2^{\prime \prime}-10^{\prime \prime}$, Imanishi et al. 2007, 2009). Imaging line surveys through interferometric observations have pushed our view of the ISM even further to show how the chemistry can provide insight into the variations of the physical processes across the central kpc of starburst (SB) galaxies (Meier \& Turner 2005, 2012). However, such studies toward active galactic nucleus (AGN) galaxies have been more limited because of the lack of sensitivity (e.g., García-Burillo et al. 2010).

The full potential of extragalactic chemistry is just emerging thanks to new generation instruments, such as ALMA and the upcoming NOEMA. In fact, within the past year and while still in the early science phase, ALMA is already providing extremely high quality data on the chemistry of some of the brightest nearby prototypical galaxies, both AGNs (NGC 1068, García-Burillo et al. 2014; Takano et al. 2014; Viti et al. 2014; NGC 1097, Izumi et al. 2013) and SBs (NGC 253, Meier et al. 2014). The unprecedented capabilities of these instruments allow us to extend molecular studies to either farther or fainter objects, thus increasing the significance of chemical comparative studies, now limited to a very reduced number of sources (Aladro et al. 2014).

The prototypical nucleus in NGC 1097 hosts the first reported low-luminosity $\left(L_{2-10 \mathrm{keV}}=4.4 \times 10^{40} \mathrm{ergs} \mathrm{s}^{-1}\right.$ Terashima et al. 2002; Nemmen et al. 2006) Seyfert 1 nucleus
(Storchi-Bergmann et al. 1993). The nuclear region is surrounded by a $\sim 700$ pc radius ring (Barth et al. 1995; Quillen et al. 1995) with ongoing star formation at a rate of $\sim 5 M_{\odot} \mathrm{yr}^{-1}$ (Hummel et al. 1987). The molecular material in NGC 1097 has been extensively studied at high resolution (Kohno et al. 2003; Hsieh et al. 2008, 2011, 2012). Molecular gas is distributed in a central concentration of $\sim 350 \mathrm{pc}$ that we refer to as the circumnuclear disk, directly surrounding the AGN, and the weaker $1.4 \mathrm{kpc}$ diameter molecular ring, the circumnuclear star forming (SF) ring (Hsieh et al. 2008).

Though located at a similar distance (14.5 Mpc, Tully 1988) than the Seyfert 2 galaxy NGC 1068, not only the nucleus of NGC 1097 does show fainter X-ray luminosity, but it is also one order of magnitude fainter both in IR luminosity (Sanders et al. 2003) and in dense gas as traced by HCN (Hsieh et al. 2012; Krips et al. 2011). The unprecedented sensitivity of ALMA allows us to carry out multimolecule imaging studies of NGC 1097 which can be directly compared to similar studies toward the much brighter NGC 1068 (Takano et al. 2014).

In our first paper, ALMA observations of $\mathrm{HCN}$ and $\mathrm{HCO}^{+}$at both $J=4-3$ and 1-0 were reported by Izumi et al. (2013). In this paper we present a multimolecule imaging study that makes use of the species that were simultaneously observed with the $J=1-0$ of $\mathrm{HCN}$ and $\mathrm{HCO}^{+}$. A companion paper will present the continuum emission in the $3 \mathrm{~mm}$ band, as well as a kinematic study of the ratios of the brightest species, while here we only discuss the overall integrated molecular emission. Our aim is to offer insight into the molecular abundance variations in the 300 pc region surrounding its Seyfert 1 nucleus, as compared to the star forming dominated circumnuclear ring at a distance of $600-800 \mathrm{pc}$ from the center.

\section{Observations}

$3 \mathrm{~mm}$ wavelength observations toward NGC 1097 were carried out as part of the ALMA Cycle 0 early science program 2011.0.00108.S (P.I. K. Kohno). Data were acquired during 2012 July 29th and October 19th with an overall on source integration time of $\sim 72 \mathrm{~min}$. The dual sideband Band 3 receivers were tuned at an LO1 frequency of $93.187 \mathrm{GHz}$. The correlator was configured in dual polarization frequency division mode (FDM) with the $4 \times 1.875 \mathrm{GHz}$ spectral windows covering the rest frequency ranges $85.8-89.4 \mathrm{GHz}$ and $97.7-101.3 \mathrm{GHz}$ (Fig. 1). The original frequency resolution of $488 \mathrm{kHz}$ was degraded by 10 channels down to a velocity resolution of $\sim 17-14.5 \mathrm{~km} \mathrm{~s}^{-1}$ across the covered frequency range.

The phase center of the observations was $\alpha_{J 2000}=$ $02^{\mathrm{h}} 46^{\mathrm{m}} 19.06^{\mathrm{s}}, \delta_{J 2000}=-30^{\circ} 16^{\prime} 29.7^{\prime \prime}$. The HPBW of the primary beam of the $12 \mathrm{~m}$ antennas is $72^{\prime \prime}-61^{\prime \prime}$ at the extremes of the frequency coverage. At the distance of $14.5 \mathrm{Mpc}$, the angular 
Table 1. Spectral parameters of detected transitions.

\begin{tabular}{lccl}
\hline \hline Molecule & Transition & $\begin{array}{c}\text { Frequency } \\
\mathrm{GHz}\end{array}$ & $\begin{array}{l}E_{\text {upper }} \\
(\mathrm{K})\end{array}$ \\
\hline $\mathrm{H}^{13} \mathrm{CN}$ & $1-0$ & $86.340^{a}$ & 4 \\
$\mathrm{H}^{13} \mathrm{CO}^{+}$ & $1-0$ & 86.754 & 4 \\
$\mathrm{SiO}$ & $2-1$ & 86.846 & 6 \\
$\mathrm{CCH}$ & $1-0$ & $87.316^{a}$ & 4 \\
$(\mathrm{HNCO})$ & $4_{1,4}-3_{1,3}$ & 87.597 & 54 \\
$\mathrm{HNCO}$ & $4_{0,4}-3_{0,3}$ & $87.925^{a}$ & 10 \\
$(\mathrm{HNCO})$ & $4_{1,3}-3_{1,2}$ & 88.239 & 54 \\
$\mathrm{HCN}$ & $1-0$ & $88.631^{a}$ & 4 \\
$\mathrm{HCO}{ }^{+}$ & $1-0$ & 89.188 & 4 \\
$\mathrm{CS}$ & $2-1$ & 97.980 & 7 \\
$\mathrm{SO}$ & $2 \mathrm{H}_{3}-1_{2}$ & 99.300 & 9 \\
$\mathrm{HC}_{3} \mathrm{~N}$ & $11-10$ & 100.076 & 29 \\
\hline
\end{tabular}

Notes. Undetected species are shown in brackets. ${ }^{(a)}$ Line multiplicity is taken into account during the spectral fit based on JPL catalog entries. We refer to the frequency of the brightest component.

to linear scale is $\sim 70 \mathrm{pc} /{ }^{\prime \prime}$, therefore our observations covered the central $5-4.3 \mathrm{kpc}$ at half power. The 24 and 31 antennas used on the first and second run, respectively, were configured up to distances of $\sim 250 \mathrm{~m}$ from the array center, thus covering uv ranges from 4.3 to $132 k \lambda(14-447 \mathrm{~m})$. This configuration resulted on an average synthesized beam of $\sim 2.2^{\prime \prime} \times 1.5^{\prime \prime}$ $(150 \mathrm{pc} \times 100 \mathrm{pc})$ with $\mathrm{PA}=100^{\circ}$.

The quasars J1924-292 and J0334-401 were used as bandpass and phase calibrator, respectively. Absolute flux scaled was calibrated through observations toward Neptune. Data were calibrated and cleaned with CASA (McMullin et al. 2007), while images were produced with GILDAS ${ }^{1}$. A final root-mean square of $\sim 0.5 \mathrm{mJy}$ in the smoothed $4.88 \mathrm{MHz}$ channels (i.e., 10 original channels) was measured in the final images.

\section{Results}

As shown by the spectrum in Fig. 1, extracted from the peak of molecular emission position (see below), the $\sim 8 \mathrm{GHz}$ frequency coverage of our observations allowed the detection of nine molecular species. For the identified features, Table 1 presents some basic spectral parameters extracted from JPL spectral line catalog (Pickett et al. 1998). Two non-detected transitions of HNCO (shown in brackets) are included as their limits will serve to set constraints to the excitation conditions (see Sect. 3.2).

Figure 2 presents the moment 0 maps of each of the detected species except for SO which does not stand out in the integrated maps (see discussion in Sect. 3.2). Moment 0 maps were integrated in the velocity range [900-1600] $\mathrm{km} \mathrm{s}^{-1}$, where the molecular emission across the whole map is detected. This range was used for all species but $\mathrm{CCH}$, for which the velocity range [600-1600] $\mathrm{km} \mathrm{s}^{-1}$ (referred to the brightest hyperfine component) was integrated in order to include the two groups of hyperfine transitions. The common logarithmic color scale in Fig. 2 allows a direct comparison of the emission of the different molecular species.

\subsection{Selected positions in AGN and SF ring}

To study the molecular abundance variations both in the circumnuclear disk around the AGN and in the star forming ring, we

\footnotetext{
http://www.iram.fr/IRAMFR/GILDAS
}

Table 2. Coordinates and line parameters of extracted spectra.

\begin{tabular}{lccll}
\hline \hline Pos. & $\begin{array}{c}\Delta \alpha \\
\left({ }^{\prime \prime}\right)\end{array}$ & $\begin{array}{l}\Delta \delta \\
\left({ }^{\prime \prime}\right)\end{array}$ & $\begin{array}{l}v_{\mathrm{LSR}} \\
\left(\mathrm{km} \mathrm{s}^{-1}\right)\end{array}$ & $\begin{array}{l}\Delta v_{1 / 2} \\
\left(\mathrm{~km} \mathrm{~s}^{-1}\right)\end{array}$ \\
\hline $\mathrm{A}$ & -0.9 & 0.9 & $1290(2)$ & $190(5)$ \\
$\mathrm{B}$ & 7.2 & 5.9 & $1276(2)$ & $65(4)$ \\
$\mathrm{C}$ & 2.3 & 9.4 & $1222.2(1.5)$ & $74.5(1.5)$ \\
$\mathrm{D}$ & -5.8 & 8.6 & $1023.1(1.0)$ & $39(2)$ \\
$\mathrm{E}$ & -9.9 & -1.3 & $1131.1(0.6)$ & $57.6(1.4)$ \\
$\mathrm{F}$ & -8.1 & -5.8 & $1252(3)$ & $91(6)$ \\
$\mathrm{G}$ & -1.8 & -8.5 & $1405.2(1.2)$ & $49(3)$ \\
$\mathrm{H}$ & 5.4 & -5.3 & $1485.9(0.8)$ & $56(2)$ \\
$\mathrm{I}$ & 8.1 & 2.7 & $1380.3(1.2)$ & $43(3)$ \\
\hline
\end{tabular}

Notes. Relative coordinates refer to the observations nominal phase center $\alpha_{J 2000}=02^{\mathrm{h}} 46^{\mathrm{m}} 19.06^{\mathrm{s}}, \delta_{J 2000}=-30^{\circ} 16^{\prime} 29.7^{\prime \prime}$. The errors derived from the fit for $v_{\mathrm{LSR}}$ and $\Delta v_{1 / 2}$ are indicated.

have selected a sample of nine positions. Table 2 lists the relative coordinates of the selected positions referred to the phase center of the observations. Position A is selected as the brightest HCN emission position in the nuclear region of the NGC 1097 and it is offset from the phase center by $\left(-0.9^{\prime \prime}, 0.9^{\prime \prime}\right)$, measured at the center of the peak flux pixel in the image (with $0.45^{\prime \prime} /$ pixel). This position matches the measured position of the nucleus at $6 \mathrm{~cm}$ $\left(\alpha_{J 2000}=02^{\mathrm{h}} 46^{\mathrm{m}} 18.96^{s}, \delta_{J 2000}=-30^{\circ} 16^{\prime} 28.9^{\prime}\right.$; Hummel et al. 1987) as well as the $860 \mu$ m continuum peak (Izumi et al. 2013). The spectra extracted at this position are shown in Fig. 1.

All other positions correspond to peaks of molecular emission of the brightest species, with the selection mainly driven by the maps of the brightest species, namely $\mathrm{HCN}, \mathrm{C}_{2} \mathrm{H}$ and $\mathrm{CS}$. Figure 3 presents the individual spectra extracted from all selected positions, both in the AGN and in the SF ring.

\subsection{Excitation and column density determination}

Rather than basing our result on direct integrated intensity line ratios, we estimated the column densities of each of the molecular species to have a more accurate determination of the molecular abundances in each position.

We used the spectral line modeling tools within the MADCUBA_IJ package ${ }^{2}$ (Martín et al in prep.) to fit the molecular emission in the spectra of the selected sample positions. The main assumption of this modeling is the emission under LTE conditions. The parameters of column density, velocity and linewidth are left as free parameters. Table 2 presents the average velocity and linewidth measured in all species. See Hsieh et al. (2011) for a discussion on the kinematics and the different linewidths measured in the star forming ring cloud complexes. However, excitation temperature and the source size parameters were fixed to $8 \mathrm{~K}$ and $1.8^{\prime \prime}$, respectively. These assumptions are explained in the following.

The gas in both the SF ring and around the AGN have kinetic temperatures higher than our assumed excitation temperature $\left(T_{\mathrm{k}}=30-90 \mathrm{~K}\right.$; Piñol-Ferrer et al. 2011). This is due to the fact that the molecular gas is subthermally excited. For $\mathrm{HCN}$ and $\mathrm{HCO}^{+}$, Izumi et al. (2013) combined the $J=1-0$ and 4-3 transitions, observed with ALMA, smoothed down to the resolution of the 3-2 transition, observed with the SMA (Hsieh et al. 2012), in order to estimate the excitation conditions of the gas traced by these species. The emission can be roughly fitted in LTE by a rotational temperature of $\sim 8 \mathrm{~K}$. This result is similar in the central

2 http://cab.inta-csic.es/madcuba/Portada.html 


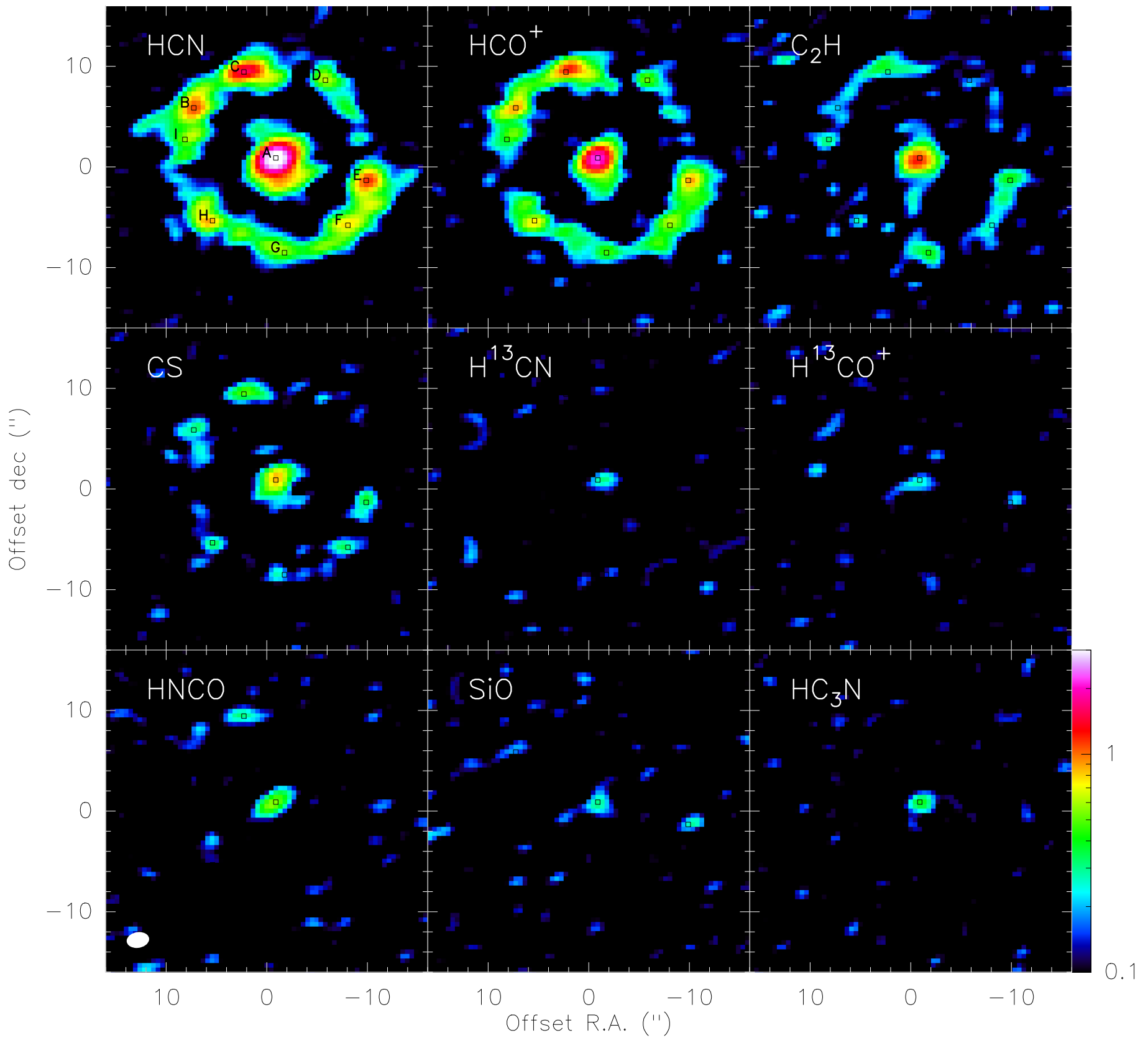

Fig. 2. Moment 0 maps of all detected species in the velocity range [900-1600] $\mathrm{km} \mathrm{s}^{-1}$, except for CCH where a range [600-1600] $\mathrm{km} \mathrm{s}^{-1} \mathrm{was}^{-1}$ used to include the two groups of hyperfine components. The $1 \sigma$ noise level in the integrated maps is $62 \mathrm{mJy} \mathrm{km} \mathrm{s}{ }^{-1}$ for all species, but for CCH which is $77 \mathrm{mJy} \mathrm{km} \mathrm{s}^{-1}$. Logarithmic color scale is set to allow a direct comparison of the emission of the different species. Color levels are set to a minimum of $0.1 \mathrm{mJy} \mathrm{km} \mathrm{s}^{-1}$ so that emission over $3 \sigma$ level is seen above the black background. Positions where spectra were extracted are marked in all boxes, and labeled in the upper left box.

position A around the AGN and toward the SF ring. Though the assumption on the excitation temperature will not strongly affect the relative abundances given that most of our observed transitions have similarly low $(4-10 \mathrm{~K})$ energies, it might be an issue for the transition of $\mathrm{HC}_{3} \mathrm{~N}$ with a significantly higher upper energy level (Table 1). However, the work by Aladro et al. (2011b) toward nearby starbursts shows that such subthermal excitation affects equally to other molecular species in their lower $J$ transitions, to those not only at lower energies but also up to transitions with $\sim 50 \mathrm{~K}$ upper energy levels. Additionally the non detection of the two higher energy transitions of HNCO listed in Table 1 and shown in Fig. 1 set a limit on the excitation temperature of this species to $T_{\mathrm{ex}}<20 \mathrm{~K}$.
Regarding the source size we assume the emission to be distributed in a region approximately equal to the synthesized beam in solid angle, that is $1.8^{\prime \prime}$ or $\sim 125 \mathrm{pc}$. A smaller emitting region would directly scale up the column density, thus increasing the effect of opacity. Our fit to the spectra results in an opacity of $\tau \sim 0.2$ in the brightest positions (A and B) for HCN. Assuming a source size of half our assumed value would increase the opacities up to $\tau \gtrsim 1$ and the derived column densities by a factor of $\sim 5$. Izumi et al. (2013) also found low opacities in the high-J transitions of HCN in the center of NGC 1097. Based on the peak line ratio of $\mathrm{HCN}$ and $\mathrm{H}^{13} \mathrm{CN}$ of $\sim 15$ and assuming a ${ }^{12} \mathrm{C} /{ }^{13} \mathrm{C}$ isotopic ratio of $\sim 40$ (Henkel et al. 2014) we can estimate an opacity of $\tau>2$ toward the central position. It could be 
S. Martín et al.: Multimolecule observations toward NGC 1097

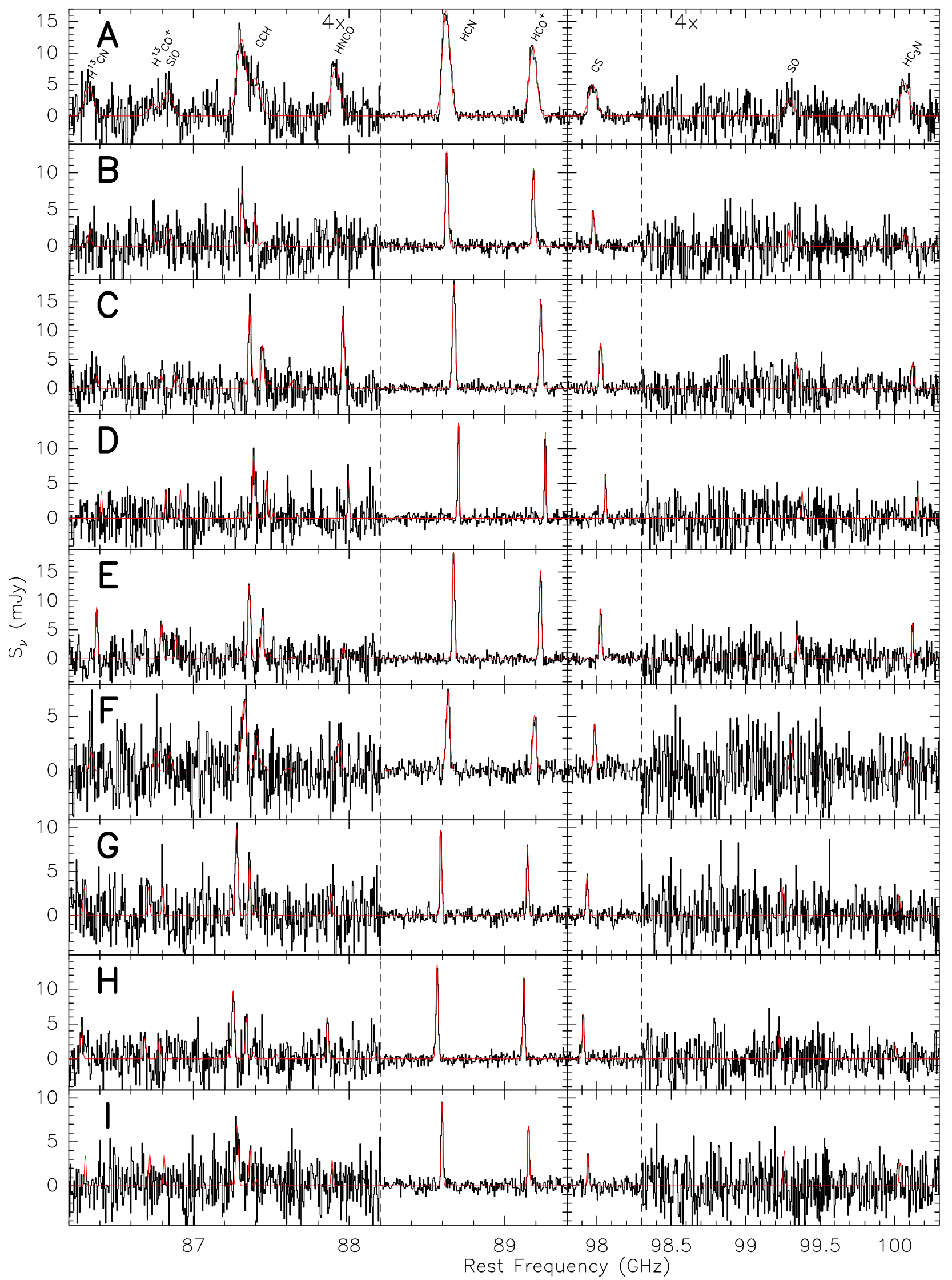

Fig. 3. Spectra extracted from selected positions in the AGN position (A) and on the SF ring (B-I) of NGC 1097. Frequency span has been cropped with respect to that of Fig. 1 removing the line free frequencies at the edges of both sidebands. Flux density scale has been multiplied by a factor of $\times 4$ in the regions (defined by the vertical dashed lines) containing the fainter lines to improve their visualization. Molecular fit to the observed emission is represented by the red line. Non-detections are presented as the $3 \sigma$ limit Gaussian profiles. 
Table 3. Column densities for each species and the selected position.

\begin{tabular}{lllllllllll}
\hline \hline Pos. & $\begin{array}{l}N_{\mathrm{HCN}} \\
10^{14} \mathrm{~cm}^{-2}\end{array}$ & $\begin{array}{l}N_{\mathrm{HCO}^{+}} \\
10^{14} \mathrm{~cm}^{-2}\end{array}$ & $\begin{array}{l}N_{\mathrm{CCH}} \\
10^{14} \mathrm{~cm}^{-2}\end{array}$ & $\begin{array}{l}N_{\mathrm{CS}} \\
10^{14} \mathrm{~cm}^{-2}\end{array}$ & $\begin{array}{l}N_{\mathrm{H}^{13} \mathrm{CN}} \\
10^{14} \mathrm{~cm}^{-2}\end{array}$ & $\begin{array}{l}N_{\mathrm{H}^{13} \mathrm{CO}^{+}} \\
10^{14} \mathrm{~cm}^{-2}\end{array}$ & $\begin{array}{l}N_{\mathrm{HNCO}} \\
10^{14} \mathrm{~cm}^{-2}\end{array}$ & $\begin{array}{l}N_{\mathrm{SiO}} \\
10^{14} \mathrm{~cm}^{-2}\end{array}$ & $\begin{array}{l}N_{\mathrm{HC} \mathrm{N}} \\
10^{14} \mathrm{~cm}^{-2}\end{array}$ & $\begin{array}{l}N_{\mathrm{SO}} \\
10^{14} \mathrm{~cm}^{-2}\end{array}$ \\
\hline A & $3.14(0.07)$ & $1.16(0.03)$ & $14.4(0.8)$ & $1.85(0.11)$ & $0.26(0.05)$ & $0.06(0.02)$ & $3.1(0.3)$ & $0.25(0.05)$ & $2.6(0.3)$ & $0.6(0.2)$ \\
B & $0.84(0.04)$ & $0.38(0.02)$ & $3.3(0.5)$ & $0.56(0.05)$ & $<0.04$ & $<0.02$ & $<0.2$ & $<0.04$ & $<0.3$ & $<0.2$ \\
C & $1.34(0.02)$ & $0.60(0.02)$ & $5.7(0.6)$ & $1.02(0.06)$ & $<0.04$ & $<0.02$ & $1.58(0.17)$ & $<0.04$ & $0.58(0.15)$ & $0.4(0.1)$ \\
D & $0.54(0.03)$ & $0.24(0.01)$ & $2.9(0.4)$ & $0.42(0.03)$ & $<0.04$ & $<0.02$ & $0.38(0.09)$ & $<0.04$ & $0.34(0.11)$ & $<0.2$ \\
E & $1.08(0.02)$ & $0.51(0.02)$ & $5.2(0.5)$ & $0.88(0.04)$ & $0.12(0.02)$ & $0.08(0.02)$ & $<0.2$ & $0.07(0.02)$ & $0.62(0.14)$ & $0.3(0.1)$ \\
F & $0.64(0.04)$ & $0.24(0.02)$ & $4.0(0.6)$ & $0.48(0.04)$ & $<0.04$ & $<0.02$ & $0.4(0.2)$ & $<0.04$ & $0.37(0.19)$ & $<0.2$ \\
G & $0.47(0.02)$ & $0.20(0.02)$ & $3.7(0.4)$ & $0.43(0.03)$ & $<0.04$ & $<0.02$ & $<0.2$ & $<0.04$ & $<0.3$ & $<0.2$ \\
H & $0.76(0.02)$ & $0.34(0.02)$ & $3.4(0.4)$ & $0.56(0.06)$ & $0.06(0.02)$ & $<0.02$ & $0.54(0.14)$ & $<0.04$ & $<0.3$ & $<0.2$ \\
I & $0.40(0.02)$ & $0.18(0.02)$ & $2.4(0.4)$ & $0.28(0.05)$ & $<0.04$ & $<0.02$ & $<0.2$ & $<0.04$ & $<0.3$ & $<0.2$ \\
\hline
\end{tabular}

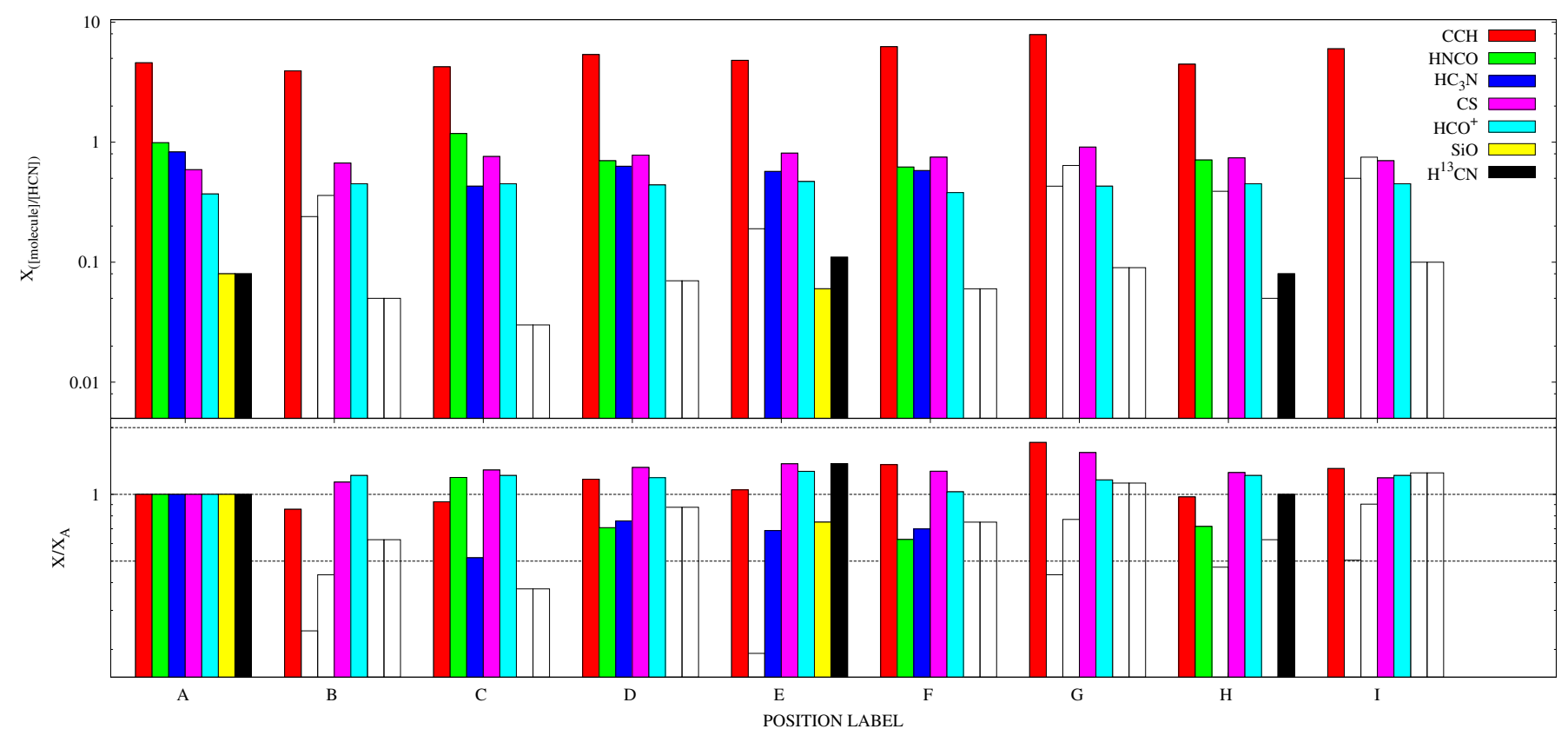

Fig. 4. Top: molecular abundances relative to HCN estimated in the sample of selected positions for all detected species. Colored histograms represent species detected while empty histograms represent upper limits to the detection. Bottom: abundances normalized to those of position A for comparison. As a reference, the three horizontal dotted lines represent equal relative abundances or a factor of two above and below those in position A.

even larger for a higher carbon isotopic ratio (Martín et al. 2010). This shows that in the central position, the HCN emission either stems from regions significantly smaller than the beam, or the carbon isotopic ratio is significantly smaller in this region. We cannot disentangle these two possibilities with our data. Though this uncertainty on the source size may have a moderate impact in the relative abundances of the brightest species, we have no further clues on the actual molecular clump source sizes across the galaxy so we assume a similar source size for all positions as a first approximation.

The derived column densities and upper limits are listed in Table 3 for all selected positions and detected species. We note that both $\mathrm{H}^{13} \mathrm{CO}^{+}$and $\mathrm{SO}$ detections are at the edge of detection. SO does not even show up in the integrated maps (Sect. 3), while the moment 0 detection of $\mathrm{H}^{13} \mathrm{CO}^{+}$might be the result of $\mathrm{SiO}$ contamination. Derived column densities for these two species are $\sim 3 \sigma$ in the few positions where those are detected, and it results in an overabundance of $\mathrm{H}^{13} \mathrm{CO}^{+}$in position $\mathrm{E}$ of a factor of $\sim 4$ which may indicate the fit to be dominated by an spurious spectral artifact (see fit in Fig. 3). Thus these species will not be included in the following discussion.

\subsection{Relative molecular abundances}

We aim to understand the chemical differentiation between the molecular gas in the SF ring and the material in the close vicinity of the nuclear engine. The central few hundred parsecs circumnuclear disk (CND) contains a significant fraction of the dense molecular gas in NGC 1097 (Table 3). Based on the integrated intensity maps of $\mathrm{HCN}$ and $\mathrm{HCO}^{+}$in Fig. 2 the central $600 \mathrm{pc}$ region encloses $32 \%$ and $25 \%$ of the total detected emission, respectively. This fraction is as much as $40 \%$ for CS or $\mathrm{CCH}$. However, we are mostly interested in the relative abundances between the different molecular species so to determine whether an AGN driven chemistry can be claimed in the central region.

We used the brightest species in our observations, namely $\mathrm{HCN}$, as a reference column density baseline. Figure 4 shows the abundances of each species relative to HCN. Molecules are displayed in decreasing order of abundance as measured in position A. To better evaluate the differences between position A and those in the SF ring, we have normalized the relative abundances to those in position A (lower panel in Fig. 4). 
The selection of a good "baseline" species for normalization is important but not critical in our study. As noted by Kohno et al. (2003), HCN is observed to be significantly enhanced toward the nucleus relative to $\mathrm{CO}$ which might point out $\mathrm{HCN}$ as a not the ideal candidate to become a reference molecule. However, this $\mathrm{HCN} / \mathrm{CO}$ trend gets less evident when higher $J$ transitions of $\mathrm{CO}$ are considered (Hsieh et al. 2012).

We see in Fig. 4 that CS is consistently slightly more abundant in the star forming ring positions. This could be caused by the normalization by $\mathrm{HCN}$, implying an slight overabundance of $\mathrm{HCN}$ in the nuclear region as previously claimed as an AGN chemical imprint. Therefore, another possibility among the brightest species would have been the use of CS, as done in previous works (Martín et al. 2009a, 2011; Aladro et al. 2011a, 2013). However, we note that reproducing Fig. 4 with the abundances relative to $\mathrm{CS}$ results in an overall underabundances of all species detected in the SF ring compared to position A. Given that, a priori, we have no reasons to assume all species to be overabundant in the CND, we stick to our use of $\mathrm{HCN}$ as it does provide abundances less biased toward the AGN position. However, this uncertainty in the reference species is taken into account in the following discussion.

Though we will deal with relative abundance among species, for completeness we used the molecular hydrogen column densities derived from CO observations by Hsieh et al. (2011) to estimate the fractional abundances relative to $\mathrm{H}_{2}$. On average the estimated fractional abundances of $\mathrm{HCN}$ would be $X(\mathrm{HCN}) \gtrsim$ $1-2 \times 10^{-9}$ both in the nuclear region and throughout the SF ring. We consider this a limit given that they use the conversion factor $X_{\mathrm{CO}}=3 \times 10^{20} \mathrm{~cm}^{-2}\left(\mathrm{~K} \mathrm{~km} \mathrm{~s}^{-1}\right)^{-1}$ which is known to be overestimating of the $\mathrm{H}_{2}$ column density in dense environments. Thus the relative abundance could be as high as $X(\mathrm{HCN}) \sim 10^{-8}$.

\section{SF ring versus $A G N$ abundances}

We find that the overall relative molecular abundances in the SF ring positions are in significantly good agreement with those in the nuclear region within less than a factor of 2 . The only exception is the underabundance of HNCO in some SF ring positions. Though we do not find enormous differences in the abundances among the selected positions, some species appear to show a differentiation between their abundances in the surroundings of the AGN and the positions in the star forming dominated ring. In particular, $\mathrm{HC}_{3} \mathrm{~N}$ and $\mathrm{HNCO}$ appear to be the species showing the largest enhancement in the nuclear region. In the following we further discuss the differences observed.

\section{1. $\mathrm{HCO}^{+}$and $\mathrm{CS}$}

The relative abundances of $\mathrm{HCO}^{+}$and $\mathrm{CS}$ derived in the individual SF ring positions are found to be consistently, but still marginally regarding the excitation uncertainties, 20-50\% above those found toward the AGN position.

These two molecules are claimed to be enhanced by UV radiation in the surroundings of star forming regions. Though its abundance may be equally enhanced by $\mathrm{X}$-ray irradiation, $\mathrm{HCO}^{+}$ is shown to be abundant in the presence of UV fields (Bayet et al. 2008, 2011) and therefore it could be associated to the massive star formation. Additionally, $\mathrm{HCO}^{+}$is also observed to be enhanced in high velocity shocked material as observed in Galactic supernovae remnants (Dickinson et al. 1980; Wootten 1981) which, again would link its enhancement to massive star formation. High abundances of CS may be understood through

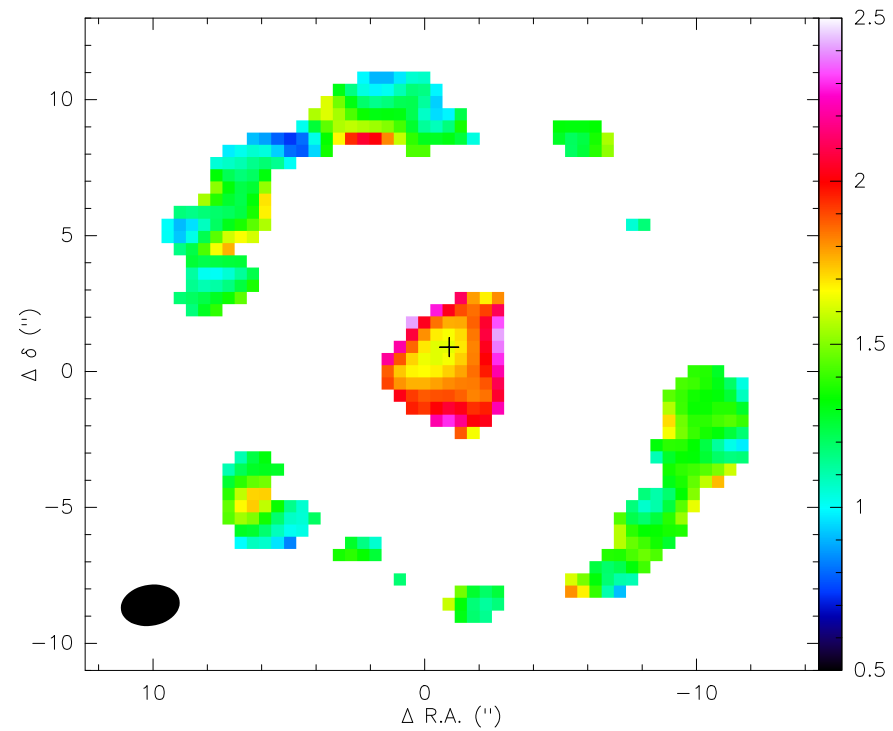

Fig. 5. Integrated intensity $\mathrm{HCN}$-to- $\mathrm{HCO}^{+}$line ratio. Values only above $5 \sigma$ in the individual integrated images has been used in this map. The position of the peak of molecular emission is shown with a cross. Synthesized beam size is represented in the bottom left corner.

the enhancement of its precursor $\mathrm{S}^{+}$in the gas phase(Drdla et al. 1989; Goicoechea et al. 2006), though there is some disagreement between theoretical models and observations (Martín et al. 2008; Tideswell et al. 2010).

Both $\mathrm{HCO}^{+}$and $\mathrm{CS}$ follow closely the emission of $\mathrm{HCN}$, and therefore they may be just probing the same dense gas. We do not observe a clear morphological differentiation with respect to $\mathrm{HCN}$ at our achieved resolution in the SF ring so as to attribute their emission to the star formation in the region. However, the significant star formation in the ring could explain their overall significantly enhanced emission with respect to the central region. We observe an $\mathrm{HCN} / \mathrm{HCO}^{+}$ratio which is higher, both in the AGN and the SF ring, than what is measured in other starburst galaxies (Krips et al. 2008). Rather than a general enhancement of HCN in this NGC 1097 due to the presence of the AGN, it is likely that in starbursting galaxies $\mathrm{HCO}^{+}$is further enhanced due to the star formation event. In such SF dominated environments, ionization due to cosmic rays accelerated in supernovae remnants (Ackermann et al. 2013) would also contribute to pull $\mathrm{HCN} / \mathrm{HCO}^{+}$down (Wootten 1981) compared to what is observe even in the moderately SF ring on NGC 1097, or similarly in NGC 1068. This scenario may as well explain why this ratio is moderately lower in the SF ring than in the very nuclear region, while the highest $\mathrm{HCN} / \mathrm{HCO}^{+}$ratio would be a local effect in the central region as discussed in the following.

\subsubsection{AGN Irradiation? $\mathrm{HCN} / \mathrm{HCO}^{+}$ratio}

For more than a decade now, it has been claimed that the X-ray irradiation in AGN dominated galaxies results in an enhancement of the HCN molecule relative to the overall molecular gas traced by $\mathrm{CO}$ or that affected by star formation traced by $\mathrm{HCO}^{+}$ (Kohno et al. 2001; Krips et al. 2008). In terms of relative abundances, we find that the $X\left(\mathrm{HCN} / \mathrm{HCO}^{+}\right)=2.7 \pm 0.1$ in the central position is only marginally larger than the values in the SF ring which range 2.1-2.7.

The high sensitivity of the ALMA data allows us to study the distribution of this ratio across the imaged region. In Fig. 5 we show $\mathrm{HCN} / \mathrm{HCO}^{+}$integrated line intensity ratio, following 
discussions in the literature. To make the most out of the high quality of the data and to avoid noise effects, we have only included points above a $5 \sigma$ level in the individual images.

Regarding its comparison with the outer SF ring, a clear $\mathrm{HCN} / \mathrm{HCO}^{+}$integrated intensity ratio enhancement is observed in the central 300 pc region of NGC 1097, as expected in an AGN dominated environment. However, this enhancement does not reach the maximum at the central position, as discussed in Sect. 5.1. It is interesting to note that the $\mathrm{HCN} / \mathrm{HCO}^{+}$measured is consistently high $(>1)$ all over the galaxy. That is, setting these values in the proposed diagnostic diagrams based on the $\mathrm{HCN} / \mathrm{HCO}^{+}$ratio (Krips et al. 2008), all the molecular gas in NGC 1097 would appear in the AGN dominated regime. The difference between the $\mathrm{HCN} / \mathrm{HCO}^{+}$in the nucleus as compared to the SF ring is less pronounced than in NGC 1068 where the ratio drops to $\sim 1$ in the ring (Viti et al. 2014). However, they only detected these species in a handful of compact positions on the SF ring, and a significant fraction of the gas in the image might be resolved out.

Extensive literature can be found where $\mathrm{HCN}$ is claimed to be enhanced by the effect of X-ray irradiation (see Graciá-Carpio et al. 2006; Krips et al. 2008, and references therein). A clear correlation is found between the $\mathrm{CO}$-normalized $\mathrm{X}$-ray flux in the $6-8 \mathrm{keV}$ band and $\mathrm{HCN}$ emission (García-Burillo et al. 2014), which is, however, also found for $\mathrm{HCO}^{+}, \mathrm{SiO}$ and $\mathrm{CN}$ (García-Burillo et al. 2010). Possibilities for the HCN enhancement such as oxygen depletion are discarded based on ancillary molecular line ratios not matching the predictions (Usero et al. 2004). The enhancement due to the presence of massive star forming regions explored by Graciá-Carpio et al. (2006) for LIRGs was discarded toward NGC 1097 in view of the low star forming rate in its nuclear region (Izumi et al. 2013). However, even the X-ray enhancement appears to be out of the question (Izumi et al. 2013) given that most models assume AGN luminosities of $L_{X} \sim 10^{43-44} \mathrm{erg} \mathrm{s}^{-1}$ (Meijerink \& Spaans 2005; Meijerink et al. 2007, 2013), which is more than two orders of magnitude higher than the values measured in NGC 1097 or NGC 1068. Nevertheless, intrinsic X-ray fluxes may be significantly higher. Such is the case of NGC 1068 where its nucleus is absorbed by $\mathrm{H}_{2}$ column density $>10^{24} \mathrm{~cm}^{-2}$ (Matt et al. 1997) and its intrinsic luminosity is estimated to be a $\sim 2$ orders of magnitude larger than observed (Iwasawa et al. 1997; Colbert et al. 2002). However, in the case of NGC 1097, the absence of Fe $\mathrm{K}$ emission lines in its X-ray spectra completely rules out the possibility of the presence of a heavily absorbed AGN revealing an intrinsically low-luminosity AGN. In such case it is not expected that the sphere of AGN influence goes beyond a few tens of parsecs in these sources. Figure 5 shows the central region of $\mathrm{HCN} / \mathrm{HCO}^{+}$enhancement to be extended well beyond one synthesized beam (see Sect. 5.1). Based on the modeling by Harada et al. (2010), Izumi et al. (2013) therefore proposed high-temperature chemistry as the key to enhance $\mathrm{HCN}$ and therefore obtain an increase in the $\mathrm{HCN} / \mathrm{HCO}^{+}$ratio in the nucleus of NGC 1097. LVG analysis by Hsieh et al. (2008) based on interferometric $\mathrm{CO} 2-1$ and 1-0 shows the presence of such high temperature gas $\left(T_{\text {kin }}>400 \mathrm{~K}\right)$ in the nuclear region. Similarly, the LVG analysis of a sample of AGN sources with enhanced $\mathrm{HCN}$ emission resulted in kinetic temperatures $T_{\mathrm{K}}>40 \mathrm{~K}$ (Krips et al. 2008) at single dish resolution. High resolution multitransition observations toward NGC 1068 show clumps in the CND with temperatures above $100 \mathrm{~K}$ (Krips et al. 2011; Viti et al. 2014). Regarding the origin of such heating, mechanical heating is suggested to explain the required high temperature in NGC 1097 (Loenen et al. 2008; Izumi et al. 2013).

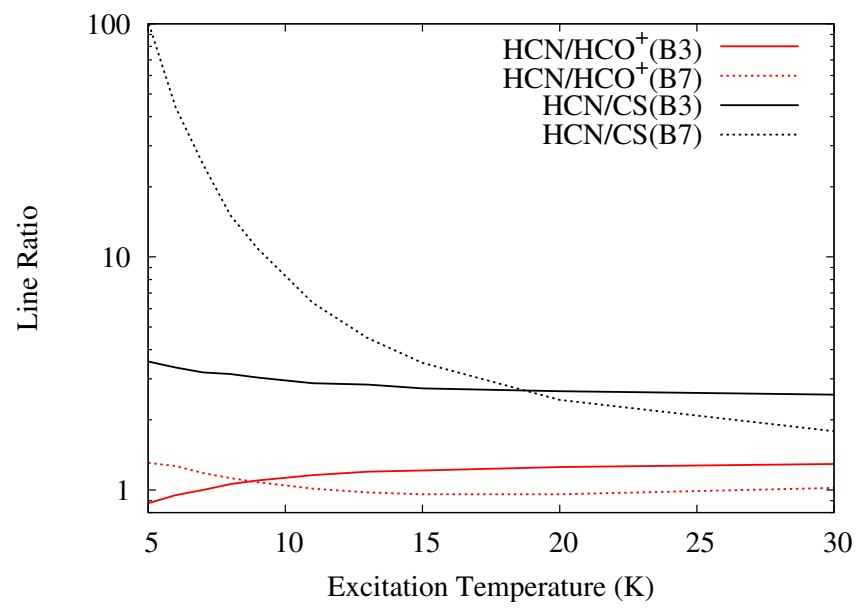

Fig. 6. $\mathrm{HCN} / \mathrm{HCO}^{+}$and $\mathrm{HCN} / \mathrm{CS}$ ratios calculated for the observable transitions in the ALMA band $3\left(\mathrm{HCN}\right.$ and $\mathrm{HCO}^{+} 1-0$, and $\left.\mathrm{CS} 2-1\right)$ and band 7 ( $\mathrm{HCN}$ and $\mathrm{HCO}^{+} J=4-3$, and CS $J=7-6$ ) as a function of the LTE excitation temperature. The curves have been calculated to reproduce the observed integrated line ratios for $T_{\mathrm{ex}}=8 \mathrm{~K}$.

A more detailed exploration of the variation of this ratio as the gas approaches the central engine is found in Sect. 5.

\subsubsection{The HCN/CS diagnostic}

Recently, Izumi et al. (2013) proposed an AGN vs. SB diagnostic diagram based on the $\operatorname{HCN}(J=4-3) / C S(J=7-6)$ ratio. Toward NGC 1097, where the CS line was not detected down to a level of $2.6 \mathrm{mJy}$, this ratio was reported to be a factor of $>3$ larger than in starburst galaxies. This difference in the line ratio in AGN versus SB galaxies has also been reported at the lower- $J$ transitions with single dish observations toward NGC 1068 compared to those in NGC 253 and M 82 (Nakajima et al. 2011; Aladro et al. 2013). Based on our 5 mJy beam ${ }^{-1}$ detection of CS $J=2-1$ and assuming the LTE at $T_{\mathrm{ex}}=$ $8 \mathrm{~K}$, the CS $J=7-6$ line would have a peak flux density of $\sim 0.6 \mathrm{mJy}^{\text {beam }}{ }^{-1}$. The fact of measuring a significantly different $\mathrm{HCN} / \mathrm{CS}$ ratio between the high- $J(>13)$ and the low- $J$ transitions $(\sim 3)$ is the result of the difference between the upper energy levels of the transitions involved in the ratios. While the HCN and CS ALMA band 3 transitions have similar upper energy levels (Table. 1), there is a significant difference between that of HCN $J=4-3(42 \mathrm{~K})$ and $\mathrm{CS} J=7-6(65 \mathrm{~K})$. Such difference makes the $\operatorname{HCN}(J=4-3) / \operatorname{CS}(J=7-6)$ ratio very sensitive to variations in the excitation temperature defining the energy levels populations. This is illustrated in Fig. 6 where we calculated the $\mathrm{HCN} / \mathrm{HCO}^{+}$and $\mathrm{HCN} / \mathrm{CS}$ line ratios for the transitions observable in the ALMA band 3 and 7 bands. While $\mathrm{HCN} / \mathrm{HCO}^{+}$ratios are basically insensitive to excitation conditions (as long as both species share the same excitation), similar to the low- $J \mathrm{HCN} / \mathrm{CS}$ ratio, the high- $J \mathrm{HCN} / \mathrm{CS}$ ratio shows a strong variation as a function of the excitation temperature. Thus the ratio among the high- $J \mathrm{HCN} / \mathrm{CS}$ is a less reliable probe to the abundance variations if the excitation conditions are not well constraint.

Rather than rejecting the diagnostic proposed by Izumi et al. (2013) based on this ratio, our result aims at understanding the origin of the large ratio difference observed between AGN and SB galaxies. Though a small abundance difference of up to a factor of two may be claimed as is suggested by the observed difference in the low- $J$ transition ratio (Aladro et al. 2013), the 
larger difference observed in the high- $J$ ratio is likely due to the excitation of CS. In starburst galaxies, the high- $J$ transitions of CS appear to be associated to a moderately higher excitation temperature gas component ( 15-30 K, Aladro et al. 2011b). This component appears to correspond to a dense molecular component $\left(\sim 10^{6} \mathrm{~cm}^{-3}\right)$. From the results in NGC 1097, we can suggest that this dense molecular component may be less prominent in AGNs, or CS might be not so efficiently produced there. However a more complete high resolution study of the excitation of the gas in NGC 1097 and NGC 1068 should help understanding the origin of this underabundance and/or underexcitation.

\subsection{Star formation: $\mathrm{CCH}$}

$\mathrm{CCH}$ is observed to vary significantly throughout the SF ring, with measured relative abundances $20 \%$ below (position B), up to a $70 \%$ above (position $\mathrm{G}$ ) those in the center. The distribution of the emission and abundances of $\mathrm{CCH}$ differs significantly from other species in NGC 1097.

Within the Galaxy this species is observed to be located in the UV irradiated cloud edges associated with massive star forming regions (Beuther et al. 2008; Walsh et al. 2010; Li et al. 2012). Its formation paths are favored in photodissociation regions (PDRs) by the reaction of $\mathrm{C}^{+}$with small hydrocarbons and additionally through photodissociation of $\mathrm{C}_{2} \mathrm{H}_{2}$ (Meier \& Turner 2005, and references therein). However UV radiation is also its main destruction path, and therefore $\mathrm{CCH}$ abundance is dependent on the evolutionary stage of the star forming event ( $\mathrm{Li}$ et al. 2012). In external galaxies the abundance of $\mathrm{CCH}$ appears not to be affected by the type of activity (Nakajima et al. 2011; Aladro et al. 2013). However, observations toward an extended sample of galaxies shown that $\mathrm{CCH}$ in AGNs may be similarly overabundant to starburst in the late stages of evolution while being different to earlier stage starbursts (Aladro et al. 2014). Overall, when the average galactic emission is observed in a sample of galaxies, a good correlation between $\mathrm{CCH}$ and $\mathrm{HCO}^{+}$is found (Martín et al. 2014). However, we do not find such correlation at GMC complex scale in our data. At high resolution, observations toward starburst galaxies (Meier \& Turner 2005, 2012; Meier et al. 2014) have shown CCH to be tracing UV irradiated clouds in the vicinity of both nuclear stars clusters and embedded star forming regions, showing large abundance variations across these galaxies. Similarly, we observe large relative abundances variations both in the nucleus and the SF ring in NGC 1097. Such variations might explain the observed homogeneity between different activity types where, when averaged over the whole few hundred parsecs of a galaxy, the local variations are smoothed out.

Figure 7 shows a comparison between the far-ultraviolet (FUV) emission from unobscured massive star formation in the SF ring of NGC 1097 (Gil de Paz et al. 2007) with our observed $\mathrm{HCN}$ and $\mathrm{CCH}$ emission. The north-eastern region, where the brighter FUV emission is observed, does not correspond to the higher relative abundance of $\mathrm{CCH}$. There (positions B and $\mathrm{C}$ ), we find a large fraction of dense well UV-shielded gas, therefore decreasing the $\mathrm{CCH}$ relative abundance with respect to $\mathrm{HCN}$, even though its absolute column density is as high as at other positions of the ring. Additionally the bright FUV from stars already formed would photodissociate $\mathrm{CCH}$. On the other hand, the position I, also highly illuminated in the north-east, has significantly less dense molecular gas, but one of the highest $\mathrm{CCH}$ relative abundances showing that both evolved (strong observed UV) and ongoing stage star forming (enhanced $\mathrm{CCH}$ )

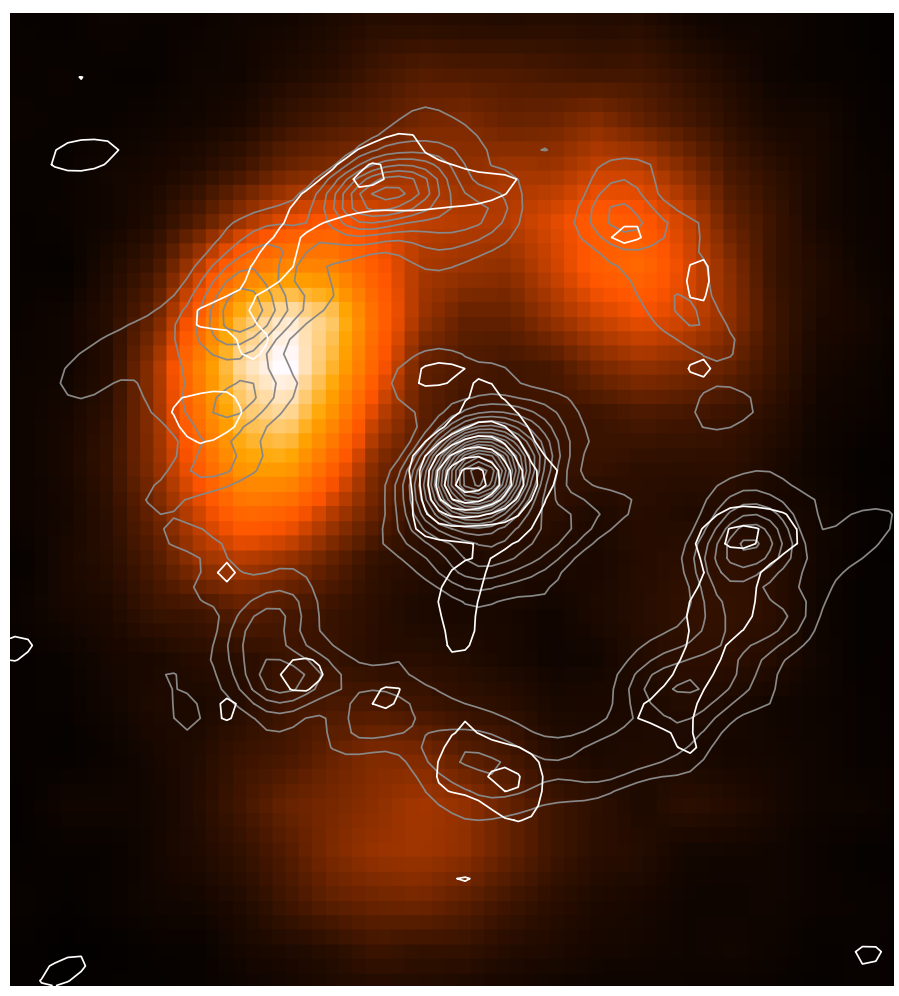

Fig. 7. Comparison between the FUV emission (in color, Gil de Paz et al. 2007), and the $\mathrm{HCN}$ (gray contours) and $\mathrm{CCH}$ (white contours).

regions co-exist in this region. That is similar to what is observed in position $G$ toward the south. There, though the observed FUV is not so intense, likely due to extinction, it is enough to photo-enhance the abundance of $\mathrm{CCH}$ in a more diffuse medium, resulting in the highest $\mathrm{CCH}$ relative abundance measured. This scenario is similar to that proposed to explain the high CCH abundance in the isolated galaxy CIG 638 (Martín et al. 2014). Finally, toward the south-west (positions E, and F), we find a region where marginal FUV is observed, again possibly due to obscuration in this gas-rich environment. If $\mathrm{CCH}$ is indeed mostly enhanced in PDRs associated with massive star forming hot cores, this would be indicative of obscured star formation in this region, which is consistent with the $860 \mu \mathrm{m}$ emission observed in these positions, also claimed to be tracing dust massive star forming regions (Izumi et al. 2013).

Though $\mathrm{CCH}$ can be a potential tracer of obscured star formation, its abundance variations can also be probing changes in the diffuseness of the ISM and therefore its permeability to the UV irradiation from massive stars. It is important to note that the critical density of $\mathrm{CCH}$ is about an order of magnitude below than that of species such as HCN so excitation effects might again play an important role in the relative abundance derived, particularly toward the densest cores.

\subsection{The densest gas: $\mathrm{SiO}, \mathrm{HNCO}$ and $\mathrm{HC}_{3} \mathrm{~N}$}

These three species are the ones showing the clearest contrast between the central region and the SF ring with a significant enhancement toward the nuclear region. Toward NGC 1068, an enhancement of $\mathrm{SiO}$ and $\mathrm{HC}_{3} \mathrm{~N}$ in the surroundings of the AGN was already been recently reported (García-Burillo et al. 2010; Takano et al. 2014).

$\mathrm{SiO}$ is only detected in one position outside the nuclear region, position $\mathrm{E}$, and there it shows a relative abundance $25 \%$ 
lower in this position. Similarly, HNCO shows an underabundance of $30-40 \%$ in the SF ring with respect to the central position, but for position $\mathrm{C}$, where HNCO shows an enhancement of $20 \%$. Moreover the limits toward the undetected positions indicate abundances $40-80 \%$ below than that in the AGN vicinity. Both species are considered as tracers of shocked dense material where shocks release both $\mathrm{SiO}$ and $\mathrm{HNCO}$ from the dust grain surfaces (Martin-Pintado et al. 1997; Huettemeister et al. 1998; Zinchenko et al. 2000; Martín et al. 2008). The emission of both species is observed to be well correlated not only in Galactic sources, but also in nearby bright galaxies (Usero et al. 2006; Meier \& Turner 2005). Surprisingly, in position E, where $\mathrm{SiO}$ has been detected in the SF ring, the lowest limit to the abundance of HNCO is found. This is likely linked to the different evolutionary stage of the embedded star forming cores where UV radiation or high-velocity shocks are destroying HNCO (Martín et al. 2008).

With a critical density of $\sim 10^{5} \mathrm{~cm}^{-3}$ (Wernli et al. 2007), $\mathrm{HC}_{3} \mathrm{~N}$ is tracing the densest regions within the GMC complexes in NGC 1097. Where detected, the measured relative abundances in the SF ring are $25-50 \%$ of that in the central position. The upper limits in the other positions are still around $40-50 \%$ below. In the case of $\mathrm{HC}_{3} \mathrm{~N}$ an increase in the temperature of the gas toward the central region would enhance this difference. Our calculated ratio of $\mathrm{HC}_{3} \mathrm{~N} / \mathrm{HCN}=1.2$ for $T_{\mathrm{ex}}=8 \mathrm{~K}$ (see Sect. 3.2) would increase by a factor of 5-6 for excitation temperatures of $\sim 20 \mathrm{~K}$.

\section{Molecular variations within the central 300 pc}

Figure 5 clearly suggests observable variations in the $\mathrm{HCN} / \mathrm{HCO}^{+}$line ratios within the CND even though the central region is only resolved over a few synthesized beams. Molecular variations in the central few hundred parsec CND around the AGN in NGC 1068 have been reported both at very high spatial resolution (35 pc, García-Burillo et al. 2014; Viti et al. 2014) for $\mathrm{HCN}$ and $\mathrm{HCO}^{+}$and at a coarser spatial resolution of $300^{\prime \prime} \times 170^{\prime \prime}$ for a wider sample of species (Takano et al. 2014). Here we discuss the different morphologies of the observed species toward NGC 1097 and compare it to what is observed in NGC 1068.

\subsection{The $\mathrm{HCN} / \mathrm{HCO}^{+}$gradient}

Within the central $300 \mathrm{pc}$ we observe that the $\mathrm{HCN} / \mathrm{HCO}^{+}$emission does not peak toward the AGN position. On the contrary, a positive gradient is observed as we get farther from the central position (Fig. 8), reaching a relatively constant maximum value of $\sim 2$. The ratio of 1.65 at the central position is still significantly higher than the average $\sim 1.3$ measured in the SF ring (Sect. 4.1.1). The relatively low $\mathrm{HCN} / \mathrm{HCO}^{+}$ratio in the close vicinity of an AGN compared to its surrounding CND has also been reported toward NGC 1068 (García-Burillo et al. 2014). Their 35 pc spatial resolution result in an even more dramatic gradient from a similar value toward the center of $\sim 1.5$ up to a ratio of $\sim 3$ in some CND regions. As discussed above when comparing with the starburst ring, this gradient could be revealing the direct effect of the AGN imprinted in the molecular abundances on its immediate surroundings. However, it has been claimed that the variation of this ratio could be the result of a density gradient, or due to differences in the opacity of these species (Meier \& Turner 2012; Meier et al. 2014).

Though $\mathrm{HCN}$ and $\mathrm{HCO}^{+}$may be moderately optically thick (see Sect. 3.2), differential opacities are likely not to play a major

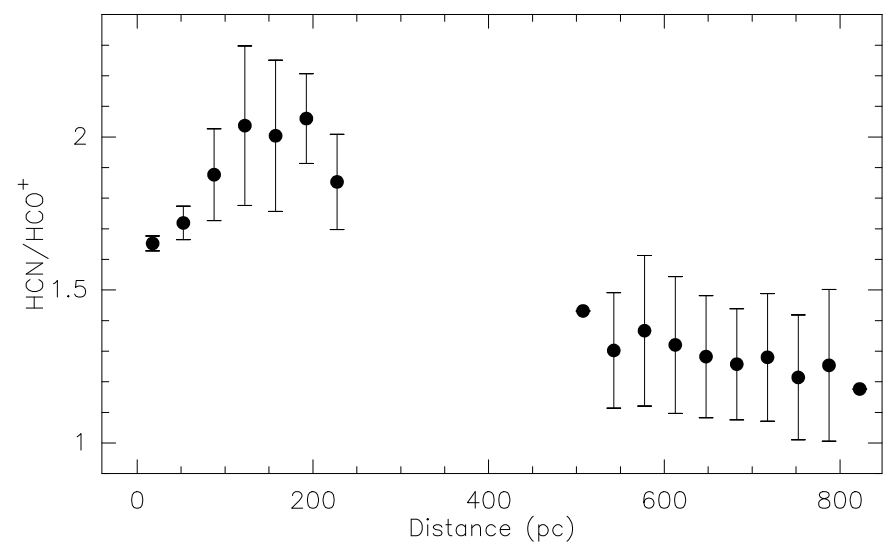

Fig. 8. Integrated intensity $\mathrm{HCN} / \mathrm{HCO}^{+}$ratio as a function of the galactocentric distance. Ratio has been averaged in bins of $0.5^{\prime \prime}(35 \mathrm{pc})$. Error bars represent the dispersion of the values on each bin.

role in the ratios observed where, for a similar column density, $\mathrm{HCO}^{+}$has an opacity almost twice that of $\mathrm{HCN}$ due to the higher radiative efficiency of $\mathrm{HCO}^{+}$as noted by Meier \& Turner (2012). Given that both species show a similar excitation temperature (Izumi et al. 2013), a differential opacity effect might result in an increased $\mathrm{HCN} / \mathrm{HCO}^{+}$ratio, where $\mathrm{HCO}^{+}$would reach saturation faster than $\mathrm{HCN}$, if both species were similarly enhanced toward the central position, contrary to what is observed. On the other hand, the difference between their critical densities might suggest that $\mathrm{HCO}^{+}$is mostly concentrated toward the nuclear region where the densities are moderate $\sim 10^{4.5} \mathrm{~cm}^{-3}$, with a higher density region ranging 100-200 pc around the AGN. This scenario is equivalent to that discussed toward local starbursts (Meier \& Turner 2012; Meier et al. 2014), though the actual density structure and morphology is likely to differ in both galaxy types.

The last scenario is a real relative abundance radial gradient from the AGN. However it could be either due to an enhancement of $\mathrm{HCN}$ production outwards from the nucleus or an inwards overproduction of $\mathrm{HCO}^{+}$. The overproduction of $\mathrm{HCO}^{+}$due to enhanced star formation is discussed in Sect. 4.1.1 and 4.2. Massive star formation could be taking place in the central $100 \mathrm{pc}$, or cosmic ray acceleration in the AGN would enhance $\mathrm{HCO}^{+}$but we have no evidence for any of these effects. Thus, we rather claim that this gradient may be understood as a result of the enhancement of $\mathrm{HCN}$ in the material surrounding the circumnuclear disk (see Sect. 5.2). Based on theoretical (Pineau des Forets et al. 1990) and Galactic observations (Tafalla et al. 2010), the enhancement of HCN in the outflow of Mrk 231 has been claimed to be the result of shock chemistry (Aalto et al. 2012) where $\mathrm{HCN}$ is efficiently formed via the reaction $\mathrm{CN}+\mathrm{H}_{2}$. We note that the $\mathrm{HCN} / \mathrm{HCO}^{+}$enhancement is observed outside the CND defined by HNCO (Sect. 5.2) and that the largest enhancement is observed in the extended HCN emission component emission. Though $\mathrm{HCO}^{+}$shows extended emission south of the central region, $\mathrm{HCN}$ appears to show an even wider component extending in the north-east and south-west directions (Fig. 2). However, we do not have other strong evidence of outflowing material in our images. On the contrary, the kinematic study of HCN by Fathi et al. (2013) shows evidence of inflowing gas.

\subsection{The CND traced by HNCO}

Even though HNCO may be one of the most fragile species in our sample, it shows an enhancement toward the central region 
(Sect. 4.3). This species is the only one clearly delineating a rotating circumnuclear disk around the AGN position as shown in Fig. 2. From the extreme emission peaks in the channel maps and taking into account the beam smearing, we measure a disk structure of $180 \pm 40 \mathrm{pc}$ length and $\mathrm{PA}=120 \pm 10^{\circ}$, covering a velocity range of $200 \mathrm{~km} \mathrm{~s}^{-1}$ around the systemic velocity. The measured velocity gradient of $1.1 \pm 0.2 \mathrm{~km} \mathrm{~s}^{-1} \mathrm{pc}^{-1}$ is consistent with that measured toward the nuclear region by Hsieh et al. (2008). The orientation of the disk is marginally smaller than the kinematic P.A. of $133^{\circ}$, and $146^{\circ}$ derived from CO 2-1 (Hsieh et al. 2011) and HCN 4-3, respectively (Fathi et al. 2013). This might indicate a real variation between the overall rotation of the gas envelope traced by $\mathrm{HCN}$ and the inner disk traced by HNCO. The possibility of HNCO tracing a bipolar outflow rather than a disk structure is unsupported by its kinematics being closely following those of $\mathrm{CO}$ and $\mathrm{HCN}$ at larger scales. As observed in other species, the south-east region of the disk is about a factor of two brighter than the north-west one. However the slightly narrower line width at this position $\left(\sim 80 \mathrm{~km} \mathrm{~s}^{-1}\right)$ makes this difference not to be apparent in the integrated maps. This is an indication of a significant asymmetrical distribution of the molecular gas in the CND (also seen in CO 2-1, Hsieh et al. 2008), similar to what is observed at higher resolution in NGC 1068 (García-Burillo et al. 2014). Also in NGC 1068, though at slightly lower resolution, the emission of HNCO is observed distributed in two lobes at both sides of the AGN (Takano et al. 2014). Such double peak distribution is in agreement with the CND being observed from a more inclined direction consistent with its Seyfert 2 nucleus.

HNCO forms in the very inner regions of molecular cloud, shielded from external UV irradiation by large visual extinction as shown by chemical models ( $A_{v}>4$ mag, Martín et al. 2009b). This species is exclusively distributed along the circumnuclear rotating structure. Within the central $300 \mathrm{pc}, \mathrm{HNCO}$ is absent in the regions outside the rotation plane of the CND, unlike the brightest species $\mathrm{HCN}, \mathrm{HCO}^{+}, \mathrm{CCH}$ and $\mathrm{CS}$, which clearly show a wider extent. In this region, low velocity shocks in the ISM due to cloud to cloud collision similar to what is observed in our Galactic center (Jones et al. 2012), as well as in external galaxies (Meier \& Turner 2005) will provide the environmental conditions for its ejection into gas phase while being dense enough to protect it from dissociation. Though sensitivity could be claimed for such non detection, the $\mathrm{HCN} / \mathrm{HNCO}$ ratio map in Fig. 9 shows already $\mathrm{HNCO}$ being exclusively concentrated in the disk, with the lower ratio toward the two CND lobes, and a clear bipolar decrease in the perpendicular direction to the plane. This fact points out to an increase of the radiation affecting the gas outside the CND. Given that star formation is not prominent in this region, UV generated in high-velocity shocks might play a significant role in the destruction of HNCO, which may also explain the enhancement of $\mathrm{HCN}$ with increasing distance from the nucleus (Sect. 5.1). Additionally, an ionization cone due to a putative jet or direct exposure to the X-ray emission from the AGN, could be claimed together to explain the absence of $\mathrm{HNCO}$ within the inner region $(<100 \mathrm{pc})$ enclosed by the $\mathrm{CND}$ and the biconical shape of the $\mathrm{HCN} / \mathrm{HNCO}$ ratio map.

\section{3. $\mathrm{HC}_{3} \mathrm{~N}$ and $\mathrm{SiO}$ in the close vicinity to the $\mathrm{AGN}$}

Both $\mathrm{HC}_{3} \mathrm{~N}$ and $\mathrm{SiO}$ are observed to be concentrated within an unresolved region (i.e., $\$ 150 \mathrm{pc}$ ) around the AGN. Such a high concentration of these molecules in the surroundings of an AGN has also been reported in NGC 1068 (García-Burillo et al. 2010; Takano et al. 2014).

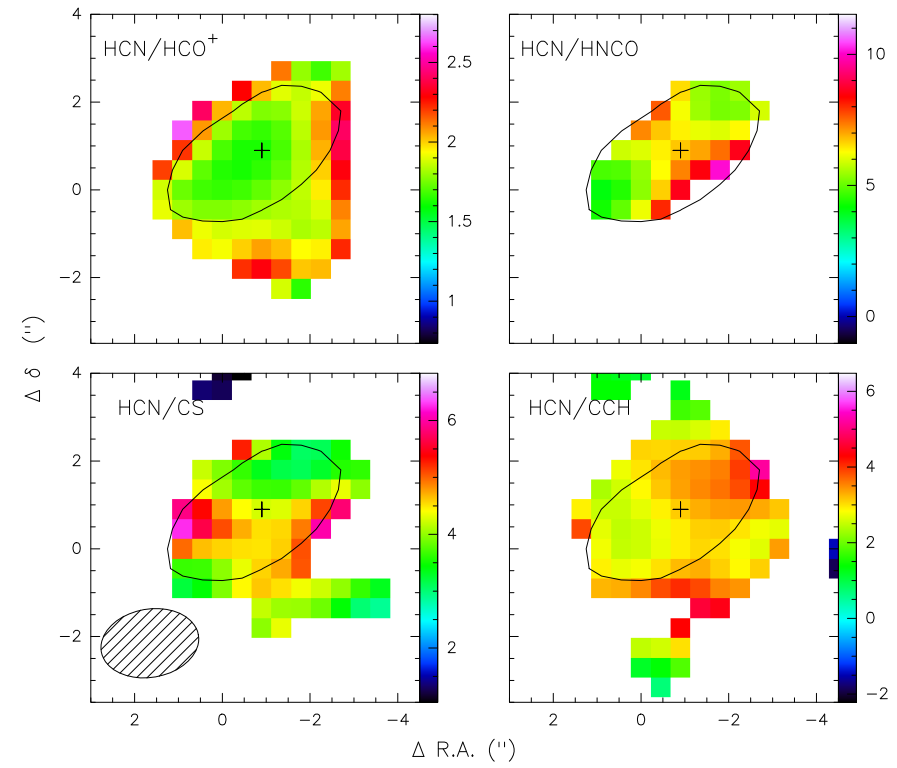

Fig. 9. Integrated intensity line ratios of $\mathrm{HCN}$ over $\mathrm{HCO}^{+}, \mathrm{HNCO}$, $\mathrm{CS}$ and $\mathrm{CCH}$ in the central $\sim 500$ pc of NGC 1097. Position of peak of molecular emission is marked with a cross. The $3 \sigma$ contour of $\mathrm{HNCO}$ emission, delineating the circumnuclear disk around the AGN, is overlaid as a reference. Synthesized beam size is represented in the bottom left box.

Though $\mathrm{HC}_{3} \mathrm{~N}$ shows some emission in the south-eastern position of the CND, the brightest in the CND, it is mostly concentrated in the central beam. Takano et al. (2014) reported that a $\sim 70 \%$ of the $\mathrm{HC}_{3} \mathrm{~N}$ emission stems from the CND in NGC 1068. Similar to what is observed in NGC $1097, \mathrm{HC}_{3} \mathrm{~N}$ is more concentrated around the AGN than molecules tracing the CND such as HNCO. Our observations together with those in NGC 1068 provide the evidence of the existence of a large amount of high density gas $\left(\sim 10^{6} \mathrm{~cm}^{-3}\right)$ shielded from the X-ray radiation from the AGN, as described by theoretical models from Harada et al. (2013). In those models, a high temperature region of the disk moderately affected by X-rays can explain an enhancement of the cyanopolyynes. In this scenario, $\mathrm{HC}_{3} \mathrm{~N}$ might be tracing the inner CND directly enclosed by HNCO. Within scales almost two orders of magnitude smaller, the few central parsecs around the Galactic black hole show $\mathrm{HC}_{3} \mathrm{~N}$ (and to a large extent $\mathrm{SiO}$ ) to be tracing the very densest clumps in the circumnuclear disk material (Martín et al. 2012).

$\mathrm{HC}_{3} \mathrm{~N}$ has been observed to be overluminous in the deeply buried IR luminous galaxy NGC 4418 (Aalto et al. 2007) which might be a signature of either buried star formation (Bayet et al. 2008) or contribution from gas in the vicinity of the AGN. $\mathrm{HC}_{3} \mathrm{~N}$ enhancement has been confirmed in other obscured LIRGs (Costagliola et al. 2011). The observed $\mathrm{HC}_{3} \mathrm{~N} / \mathrm{HCO}^{+}$integrated intensity ratio toward NGC 4418 is $\sim 0.4-0.6$ (Aalto et al. 2007; Costagliola et al. 2011). This is using the $J=10-9$ transition of $\mathrm{HC}_{3} \mathrm{~N}$, and therefore the ratio would be higher if using the $J=11-10$ in our work. The ratio we derive toward the nucleus of NGC 1097 is however $\sim 0.17$, and therefore significantly lower than the value in NGC 4418. Similarly, for those LIRGs where $\mathrm{HC}_{3} \mathrm{~N}$ was detected in the sample from Costagliola et al. (2011), a ratio ranging $\mathrm{HC}_{3} \mathrm{~N} / \mathrm{HCN} \sim 0.4-0.2$ is found (again using the fainter $J=10-9 \mathrm{HC}_{3} \mathrm{~N}$ transition). Toward NGC 1097 we observe a lower ratio of $\sim 0.1$. Though we know that both species are not coexistent in NGC 1097, and thus a higher ratio of $\mathrm{HC}_{3} \mathrm{~N}$ might be measured toward the central position, a similar non-coexistence applies in the obscured LIRGs observed 
with single-dish resolution. Therefore, based on our observations, an enhancement of $\mathrm{HC}_{3} \mathrm{~N}$ in the $\mathrm{CND}$ around a putative AGN cannot be the only contribution to the observed $\mathrm{HC}_{3} \mathrm{~N}$ enhancement in obscured LIRGs. This gives a further support to the idea of buried young star formation in this objects as a key ingredient driving their luminosities (Aalto et al. 2007; Costagliola et al. 2011). We note, however, that the line ratios $\mathrm{HC}_{3} \mathrm{~N} / \mathrm{HCO}^{+} \sim 0.16$ and $\mathrm{HC}_{3} \mathrm{~N} / \mathrm{HCN} \sim 0.1$ measured toward the close IR bright quasar, Mrk 231, are in quite good agreement to those measured in the center of NGC 1097, even though the strong star formation in Mrk 231 (Aalto et al. 2012, and references therein).

On the other hand, in the CND region $\mathrm{SiO}$ is exclusively detected toward the central beam. Though the signal to noise of our data is not high, the $\mathrm{SiO} / \mathrm{H}^{13} \mathrm{CO}^{+}>1$ line ratio is consistent with the observations toward NGC 1068 (Usero et al. 2004). $\mathrm{SiO}$ is a tracer of shocks both in the Galaxy (Martin-Pintado et al. 1992, 1997; Huettemeister et al. 1998) and in external galaxies (García-Burillo et al. 2000; Usero et al. 2006). Toward NGC 1068, X-ray and cosmic ray chemistry has been claimed to explain the $\mathrm{SiO}$ abundance in its CND (García-Burillo et al. 2010; Aladro et al. 2013) based on the correlation between $\mathrm{SiO}$ emission and the $\mathrm{Fe} 6.4 \mathrm{keV}$ line in the Galactic center (Martín-Pintado et al. 2000; Amo-Baladrón et al. 2009). However, the high abundance of the easily destroyed $\mathrm{HC}_{3} \mathrm{~N}$ would not resist photodissociation in such environment. Still, the emission could be enhanced by the $\mathrm{X}$-ray radiation in a region different to that where $\mathrm{HC}_{3} \mathrm{~N}$ is formed (Harada et al. 2013), which might explain the enhanced abundance of both species. The signal-to-noise ratio of our $\mathrm{SiO}$ detection prevents us from reaching further conclusions, but to confirm the compact $\mathrm{SiO}$ emission in the surroundings of $\mathrm{AGN}$, similar to what is observed in NGC 1068 (García-Burillo et al. 2010).

\section{4. $\mathrm{CS}$ and $\mathrm{CCH}$}

As shown in Fig. 9, the behavior of these two species in the central few hundred parsecs appear to anticorrelate. Though the resolution is limited, based on previous studies and theoretical models we suggest that such differentiation is likely to be due to real abundance differences caused by environmental conditions.

CS is observed to be evenly distributed across the CND, with a relative abundance drop of almost a factor of two in the southeastern CND region (observed peak of HCN/CS ratio in Fig. 9), contrary to what is observed in $\mathrm{CCH}$. Though its abundance varies, CS seems to be abundant in the CND and not prominent in the region outside that plane.

Our observations show the $\mathrm{CCH}$ to be more abundant (lower $\mathrm{HCN} / \mathrm{CCH}$ ratio in Fig. 9) toward the south-east CND region where the peak line emission is found for most species, with a significant but not too prominent $(\sim 60 \%)$ relative abundance decrease toward the north-western part of the CND. In addition its abundance is also significant outside of the CND plane defined by HNCO, which might be linked to outflowing material similar to what is observed in Maffei 2 (Meier \& Turner 2012). The differences observed might be due to temperature affecting its chemistry. The models from Harada et al. (2013) (for the smaller XDR fraction to better represent this low-luminosity AGN) show $\mathrm{CCH}$ abundance to decrease as the temperature increase.

\section{Summary}

In this paper we have presented the $3 \mathrm{~mm}$ molecular observations carried out with ALMA toward NGC 1097. The detection of eight different molecular species has been discussed under our current understanding of the formation and excitation of each of these species. We have developed a molecular scenario of NGC 1097 first between the star forming ring and the nuclear region and secondly regarding the barely resolved morphology of the emission of these molecules in the central $\sim 300 \mathrm{pc}$.

The highest concentration of dense gas is located in the surroundings of the AGN (Hsieh et al. 2012; Izumi et al. 2013). The species $\mathrm{HCN}, \mathrm{HCO}^{+}$and $\mathrm{CS}$ appear to be tracing the same molecular component. An increase of the abundance of $\mathrm{HCO}^{+}$ and CS relative to $\mathrm{HCN}$ of $20-50 \%$ in the star forming ring with respect to the central region is observed. The claimed large discrepancy in the HCN/CS ratio between SB and AGN galaxies (Izumi et al. 2013) is likely due to a real underabundance of CS of a factor of $\sim 2$ together with excitation differences. CS is observed to be excited to higher temperatures (Aladro et al. 2011b), which may be the evidence of a more prominent high density component $\left(10^{6} \mathrm{~cm}^{-3}\right)$ in SBs and may be less significant in AGNs. Additionally, CS could be underproduced in this high density regions in AGNs.

$\mathrm{CCH}$ shows overabundances of up to $70 \%$ in the SF ring, and its abundance distribution differs significantly from the other species. We claim it to be closely related to star formation and it might be a good tracer of obscured star formation in the ring. The largest abundance contrast is shown by the densest gas tracers, namely $\mathrm{SiO}, \mathrm{HNCO}$, and $\mathrm{HC}_{3} \mathrm{~N}$, which are clearly overabundant around the AGN.

Within the central $\sim 300 \mathrm{pc}$ HNCO clearly delineates a circumnuclear disk of $180 \pm 40 \mathrm{pc}$ around the nucleus. The disk $\mathrm{PA}=120 \pm 10^{\circ}$ is slightly less inclined than the rotation plane of the overall molecular gas in the central region $\left(\mathrm{PA}=133-146^{\circ}\right.$, Hsieh et al. 2011; Fathi et al. 2013). This disk appear to enclose the emission of $\mathrm{HC}_{3} \mathrm{~N}$ and $\mathrm{SiO}$ which suggest an stratification of the disk as predicted by models (Harada et al. 2013) with each species being formed/enhanced at different regions within the disk. In this scenario, HNCO would trace the core of the molecular clouds in the disk well protected from the radiation from the AGN. Inside we would find a dense region moderately exposed to $\mathrm{X}$-ray radiation where $\mathrm{HC}_{3} \mathrm{~N}$ would be enhanced. Finally the radiation resilient $\mathrm{SiO}$ might be tracing the innermost molecular region of the circumnuclear disk.

$\mathrm{HCN} / \mathrm{HCO}^{+}$shows a positive gradient as we move away from the AGN, similar to what is observed toward NGC 1068, and therefore an enhancement of $\mathrm{HCN}$ via X-ray from the AGN is discarded. Unless $\mathrm{HCO}^{+}$has some mechanism to be enhanced in the close vicinity of the AGN, we find plausible the HCN enhancement off the circumnuclear disk plane due to shocked gas in outflowing material from the central engine.

The results presented in this paper toward the low luminosity AGN in NGC 1097 are in agreement with previous results on the luminous AGN on NGC 1068 (García-Burillo et al. 2014; Takano et al. 2014), thus the difference in luminosity between the two sources does not appear to have a impact on its chemical structure around their AGNs. These early science ALMA results clearly show the potential of chemical studies to understand the physical processes in the circumnuclear disks around AGNs.

Acknowledgements. This paper makes use of the following ALMA data: ADS/JAO.ALMA\#2011.0.00108.S. ALMA is a partnership of ESO (representing its member states), NSF (USA), and NINS (Japan), together with NRC (Canada) and NSC and ASIAA (Taiwan), in cooperation with the Republic of Chile. The Joint ALMA Observatory is operated by ESO, AUI/NRAO, and NAOJ. 


\section{References}

Aalto, S., Monje, R., \& Martín, S. 2007, A\&A, 475, 479

Aalto, S., Garcia-Burillo, S., Muller, S., et al. 2012, A\&A, 537, A44

Ackermann, M., Ajello, M., Allafort, A., et al. 2013, Science, 339, 807

Aladro, R., Martín, S., Martín-Pintado, J., et al. 2011a, A\&A, 535, A84

Aladro, R., Martín-Pintado, J., Martín, S., Mauersberger, R., \& Bayet, E. 2011b, A\&A, 525, A89

Aladro, R., Viti, S., Bayet, E., et al. 2013, A\&A, 549, A39

Aladro, R., Martín, S., Riquelme, D., et al. 2014, A\&A, submitted

Amo-Baladrón, M. A., Martín-Pintado, J., Morris, M. R., Muno, M. P., \&

Rodríguez-Fernández, N. J. 2009, ApJ, 694, 943

Amo-Baladrón, M. A., Martín-Pintado, J., \& Martín, S. 2011, A\&A, 526, A54

Barth, A. J., Ho, L. C., Filippenko, A. V., \& Sargent, W. L. 1995, AJ, 110, 1009

Bayet, E., Viti, S., Williams, D. A., \& Rawlings, J. M. C. 2008, ApJ, 676, 978

Bayet, E., Williams, D. A., Hartquist, T. W., \& Viti, S. 2011, MNRAS, 414, 1583

Beuther, H., Semenov, D., Henning, T., \& Linz, H. 2008, ApJ, 675, L33

Colbert, E. J. M., Weaver, K. A., Krolik, J. H., Mulchaey, J. S., \& Mushotzky, R. F. 2002, ApJ, 581, 182

Costagliola, F., Aalto, S., Rodriguez, M. I., et al. 2011, A\&A, 528, A30

Davis, T. A., Heiderman, A., Evans, N. J., \& Iono, D. 2013, MNRAS, 436, 570

Dickinson, D. F., Rodriguez Kuiper, E. N., Dinger, A. S. C., \& Kuiper, T. B. H 1980, ApJ, 237, L43

Drdla, K., Knapp, G. R., \& van Dishoeck, E. F. 1989, ApJ, 345, 815

Fathi, K., Lundgren, A. A., Kohno, K., et al. 2013, ApJ, 770, L27

García-Burillo, S., Martín-Pintado, J., Fuente, A., \& Neri, R. 2000, A\&A, 355, 499

García-Burillo, S., Usero, A., Fuente, A., et al. 2010, A\&A, 519, A2

García-Burillo, S., Combes, F., Usero, A., et al. 2014, A\&A, 567, A125

Gil de Paz, A., Boissier, S., Madore, B. F., et al. 2007, ApJS, 173, 185

Goicoechea, J. R., Pety, J., Gerin, M., et al. 2006, A\&A, 456, 565

Graciá-Carpio, J., García-Burillo, S., Planesas, P., \& Colina, L. 2006, ApJ, 640, L135

Harada, N., Herbst, E., \& Wakelam, V. 2010, ApJ, 721, 1570

Harada, N., Thompson, T. A., \& Herbst, E. 2013, ApJ, 765, 108

Henkel, C., Asiri, H., Ao, Y., et al. 2014, A\&A, 565, A3

Hsieh, P.-Y., Matsushita, S., Lim, J., Kohno, K., \& Sawada-Satoh, S. 2008, ApJ, 683, 70

Hsieh, P.-Y., Matsushita, S., Liu, G., et al. 2011, ApJ, 736, 129

Hsieh, P.-Y., Ho, P. T. P., Kohno, K., Hwang, C.-Y., \& Matsushita, S. 2012, ApJ, 747, 90

Huettemeister, S., Dahmen, G., Mauersberger, R., et al. 1998, A\&A, 334, 646

Hummel, E., van der Hulst, J. M., \& Keel, W. C. 1987, A\&A, 172, 32

Imanishi, M., Nakanishi, K., Tamura, Y., Oi, N., \& Kohno, K. 2007, AJ, 134, 2366

Imanishi, M., Nakanishi, K., Tamura, Y., \& Peng, C.-H. 2009, AJ, 137, 3581

Iwasawa, K., Fabian, A. C., \& Matt, G. 1997, MNRAS, 289, 443

Izumi, T., Kohno, K., Martín, S., et al. 2013, PASJ, 65, 100

Jones, P. A., Burton, M. G., Cunningham, M. R., et al. 2012, MNRAS, 419, 2961

Knudsen, K. K., Walter, F., Weiss, A., et al. 2007, ApJ, 666, 156

Kohno, K., Matsushita, S., Vila-Vilaró, B., et al. 2001, in The Central Kiloparsec of Starbursts and AGN: The La Palma Connection, eds. J. H. Knapen,

J. E. Beckman, I. Shlosman, \& T. J. Mahoney, ASP Conf. Ser., 249, 672

Kohno, K., Ishizuki, S., Matsushita, S., Vila-Vilaró, B., \& Kawabe, R. 2003, PASJ, 55, L1

Krips, M., Neri, R., García-Burillo, S., et al. 2008, ApJ, 677, 262

Krips, M., Martín, S., Eckart, A., et al. 2011, ApJ, 736, 37

Li, J., Wang, J., Gu, Q., Zhang, Z.-y., \& Zheng, X. 2012, ApJ, 745, 47

Loenen, A. F., Spaans, M., Baan, W. A., \& Meijerink, R. 2008, A\&A, 488, L5

Martín, S. 2011, in IAU Symp. 280, eds. J. Cernicharo, \& R. Bachiller, 351
Martín, S., Mauersberger, R., Martín-Pintado, J., Henkel, C., \& García-Burillo, S. 2006, ApJS, 164, 450

Martín, S., Requena-Torres, M. A., Martín-Pintado, J., \& Mauersberger, R. 2008, ApJ, 678, 245

Martín, S., Martín-Pintado, J., \& Mauersberger, R. 2009a, ApJ, 694, 610

Martín, S., Martín-Pintado, J., \& Viti, S. 2009b, ApJ, 706, 1323

Martín, S., Aladro, R., Martín-Pintado, J., \& Mauersberger, R. 2010, A\&A, 522, A62

Martín, S., Krips, M., Martín-Pintado, J., et al. 2011, A\&A, 527, A36

Martín, S., Martín-Pintado, J., Montero-Castaño, M., Ho, P. T. P., \& Blundell, R. 2012, A\&A, 539, A29

Martín, S., Verdes-Montenegro, L., Aladro, R., et al. 2014, A\&A, 563, L6

Martin-Pintado, J., Bachiller, R., \& Fuente, A. 1992, A\&A, 254, 315

Martin-Pintado, J., de Vicente, P., Fuente, A., \& Planesas, P. 1997, ApJ, 482, L45

Martín-Pintado, J., de Vicente, P., Rodríguez-Fernández, N. J., Fuente, A., \& Planesas, P. 2000, A\&A, 356, L5

Matt, G., Guainazzi, M., Frontera, F., et al. 1997, A\&A, 325, L13

McMullin, J. P., Waters, B., Schiebel, D., Young, W., \& Golap, K. 2007, in Astronomical Data Analysis Software and Systems XVI, eds. R. A. Shaw, F. Hill, \& D. J. Bell, ASP Conf. Ser., 376, 127

Meier, D. S., \& Turner, J. L. 2005, ApJ, 618, 259

Meier, D. S., \& Turner, J. L. 2012, ApJ, 755, 104

Meier, D. S., Walter, F., Bolatto, A. D. et al. 2014, ApJ, submitted

Meijerink, R., \& Spaans, M. 2005, A\&A, 436, 397

Meijerink, R., Spaans, M., \& Israel, F. P. 2007, A\&A, 461, 793

Meijerink, R., Spaans, M., Kamp, I., et al. 2013, J. Phys. Chem. A, 117, 9593

Muller, S., Beelen, A., Guélin, M., et al. 2011, A\&A, 535, A103

Muller, S., Combes, F., Guélin, M., et al. 2014, A\&A, 566, A112

Nakajima, T., Takano, S., Kohno, K., \& Inoue, H. 2011, ApJ, 728, L38

Nemmen, R. S., Storchi-Bergmann, T., Yuan, F., et al. 2006, ApJ, 643, 652

Pickett, H. M., Poynter, I. R. L., Cohen, E. A., et al. 1998, J. Quant. Spectr. Rad. Transf., 60, 883

Pineau des Forets, G., Roueff, E., \& Flower, D. R. 1990, MNRAS, 244, 668

Piñol-Ferrer, N., Fathi, K., Lundgren, A., \& van de Ven, G. 2011, MNRAS, 414, 529

Quillen, A. C., Frogel, J. A., Kuchinski, L. E., \& Terndrup, D. M. 1995, AJ, 110, 156

Sanders, D. B., Mazzarella, J. M., Kim, D., Surace, J. A., \& Soifer, B. T. 2003 AJ, 126, 1607

Schinnerer, E., Weiß, A., Aalto, S., \& Scoville, N. Z. 2010, ApJ, 719, 1588

Storchi-Bergmann, T., Baldwin, J. A., \& Wilson, A. S. 1993, ApJ, 410, L11

Tafalla, M., Santiago-García, J., Hacar, A., \& Bachiller, R. 2010, A\&A, 522, A91

Takano, S., Nakajima, T., Kohno, K., et al. 2014, PASJ, 66, 75

Terashima, Y., Iyomoto, N., Ho, L. C., \& Ptak, A. F. 2002, ApJS, 139, 1

Tideswell, D. M., Fuller, G. A., Millar, T. J., \& Markwick, A. J. 2010, A\&A, 510, A85

Tully, R. B. 1988, Nearby galaxies catalog

Usero, A., García-Burillo, S., Fuente, A., Martín-Pintado, J., \& RodríguezFernández, N. J. 2004, A\&A, 419, 897

Usero, A., García-Burillo, S., Martín-Pintado, J., Fuente, A., \& Neri, R. 2006, A\&A, 448, 457

Viti, S., García-Burillo, S., Fuente, A., et al. 2014, A\&A, 570, A28

Walsh, A. J., Thorwirth, S., Beuther, H., \& Burton, M. G. 2010, MNRAS, 404, 1396

Wang, M., Henkel, C., Chin, Y., et al. 2004, A\&A, 422, 883

Watanabe, Y., Sakai, N., Sorai, K., \& Yamamoto, S. 2014, ApJ, 788, 4

Wernli, M., Wiesenfeld, L., Faure, A., \& Valiron, P. 2007, A\&A, 464, 1147

Wootten, A. 1981, ApJ, 245, 105

Zinchenko, I., Henkel, C., \& Mao, R. Q. 2000, A\&A, 361, 1079 